«Mon parent, ce nouvel ennemi ». Relations matrimoniales et intériorisation de l'Autre chez les Jebero d'Amazonie péruvienne

\title{
Ronan Julou
}

\section{(2) OpenEdition}

\section{Journals}

Édition électronique

URL : https://journals.openedition.org/jsa/10726

DOI : 10.4000/jsa.10726

ISSN : 1957-7842

Éditeur

Société des américanistes

Édition imprimée

Date de publication : 23 juillet 2009

Pagination : 7-39

ISSN : 0037-9174

Référence électronique

Ronan Julou, « «Mon parent, ce nouvel ennemi ». Relations matrimoniales et intériorisation de l'Autre chez les Jebero d'Amazonie péruvienne », Journal de la Société des américanistes [En ligne], 95-1 | 2009, mis en ligne le 10 juin 2014, consulté le 03 septembre 2022. URL : http://journals.openedition.org/jsa/ 10726 ; DOI : https://doi.org/10.4000/jsa.10726 


\title{
«MON PARENT, CE NOUVEL ENNEMI ». RELATIONS MATRIMONIALES ET INTÉRIORISATION DE L'AUTRE CHEZ LES JEBERO D'AMAZONIE PÉRUVIENNE
}

\author{
Ronan JULOU *
}

Les Jebero, anciens « indiens chrétiens » modèles durant l'époque coloniale, s'estiment aujourd'hui confrontés à une transformation délétère de leurs relations sociales, marquées par une perte de confiance envers leurs « parents proches », considérés comme des ennemis potentiels. Les Jebero attribuent cette déstructuration de leur cohésion sociale à la multiplication, ces dernières décennies, des mariages avec des gens extérieurs au village, incarnations d'une altérité prédatrice désormais "intériorisée ». L'auteur montre cependant que les tensions actuelles ne sont pas liées à l'identité de ces nouveaux venus, mais à l'évolution récente des relations entre alliés au sein même du village. Les accusations qui émaillent les relations entre co-résidents révèlent un changement sensible de leur rapport à la notion même d'affinité : l'allié jebero, autrefois progressivement assimilé à un consanguin, conserve à présent ses distances et rend tangible son altérité. Une conception nouvelle de l'alliance qui, comme le propose l'auteur en conclusion, peut donner sens aux revendications identitaires récemment développées par cette société face à l'État péruvien. [Mots-clés : Amazonie péruvienne, Jebero, parenté, altérité, mythologie.]

My kin, this new enemy. Matrimonial relations and internalization of the Other among the Jebero of the Peruvian Amazon. The Jebero, formerly considered model "Christian Indians » during the colonial period, are facing dire changes in their social relationships. Instead of trusting their close kin, they now see them as potential enemies. The Jebero consider this alteration of their social cohesion as due to the internalization of predatory alterity brought about by an increasing number of marriages with outsiders from other villages. Yet, I show that current tensions derive less from these outsiders' identity than from recent changes in affinal relationships within the village itself. Mutual accusations and conflicts reveal an underlying transformation of their conception of affinity: affines, who were progressively absorbed by consanguinity, now remain distant and overtly display their otherness. I conclude that this new conception of aftinity may add extra meaning to the Jebero's recent claims to the Peruvian State for

* Chercheur aftilié au Laboratoire d'anthropologie sociale, 52 rue du Cardinal Lemoine, 75005 Paris [rjulou@hotmail.com].

Journal de la Société des Américanistes, 2009, 95-1, pp. 7-39. C Société des Américanistes. 
the recognition of their native origins and attached rights. [Key words: Peruvian Amazon, Jebero, kinship, alterity, mythology.]

" Mi pariente, este nuevo enemigo ». Relaciones matrimoniales e interiorización del Otro entre los jeberos de la Amazonia peruana. Los jeberos, antiguos « indios cristianos » ejemplares durante la época colonial, se consideran hoy en día confrontados a una transformacion deleteréa de sus relaciones sociales, marcada por una pérdida de confianza hacia sus "parientes cercanos", actualmente percibidos como enemigos potenciales. Los jeberos atribuyen esta deterioración de su cohesión social a la multiplicación, durante estas últimas décadas, de matrimonios con gente de afuera, encarnaciones de una alteridad predadora actualmente interiorizada. El autor muestra sin embargo que las tensiones actuales no son vinculadas a la identidad de esos nuevos afinos sino a la evolución reciente de las relaciones entre aliados dentro del pueblo jebero. La multiplicación de las acusaciones mutuas revelan fundamentalmente una transformación de su percepción de la afinidad : el afino, antes progresivamente asimilado a un consanguino, conserva hoy en día su distancia y manifiesta su alteridad. Se trata de una nueva concepción de la alianza la cual, como lo propone el autor en la conclusión, puede dar sentido a sus recientes revendicaciones como pueblo indígena frente al Estado peruano. [Palabras claves: Amazonia peruana, jeberos, parentesco, alteridad, mitología.]

Mon père et ses deux gendres voulaient maintenant repartir, mais son frère insista pour leur donner un " cachito " [boisson à base d'alcool de canne] et à ce moment-là mon père, en buvant, attrapa le mal qui s'installa dans sa gorge. [...] Un chamane l'examina et se mit à rire. [...] Il dit alors à mon papa : « c'est ton propre frère qui t'a envoyé ce mal. [...] Tu es foutu maintenant ». Tu vois comment peuvent être les gens. À son propre frère! Et c'est comme ça jusqu'à maintenant.

Très ému, Abraham Chota dut s'interrompre à plusieurs reprises au moment d'évoquer la mort de son père. Plus encore que le souvenir d'un deuil, vécu alors qu'il n'était qu'un enfant, c'est l'identité du meurtrier présumé qui donnait à sa tragédie familiale toute sa dimension douloureuse : « son propre frère ! »

Au cours de mon séjour à Jeberos ${ }^{1}$, petit village de l'Amazonie péruvienne (Figure 1), il m'a souvent été donné d'écouter des récits où les accusations mutuelles de sorcellerie, d'infidélité et de trahison semblaient caractériser les relations entre proches parents. Récits dont la conclusion était sans appel : à Jeberos, depuis quelques décennies, il n'était plus possible de faire confiance à ses propres parents. Depuis quelques décennies seulement, car tous mes interlocuteurs achevaient leurs récriminations en rappelant de façon lancinante : " Avant, ce n'était pas comme cela ! ".

Les Jebero faisaient, par là, référence à une époque mentionnée dans la tradition orale où le village de leurs ancêtres, nouvel Eden sur terre, respirait l'harmonie. La sorcellerie était inconnue et les tensions entre villageois non seulement inexistantes, mais foncièrement contradictoires avec la notion même 


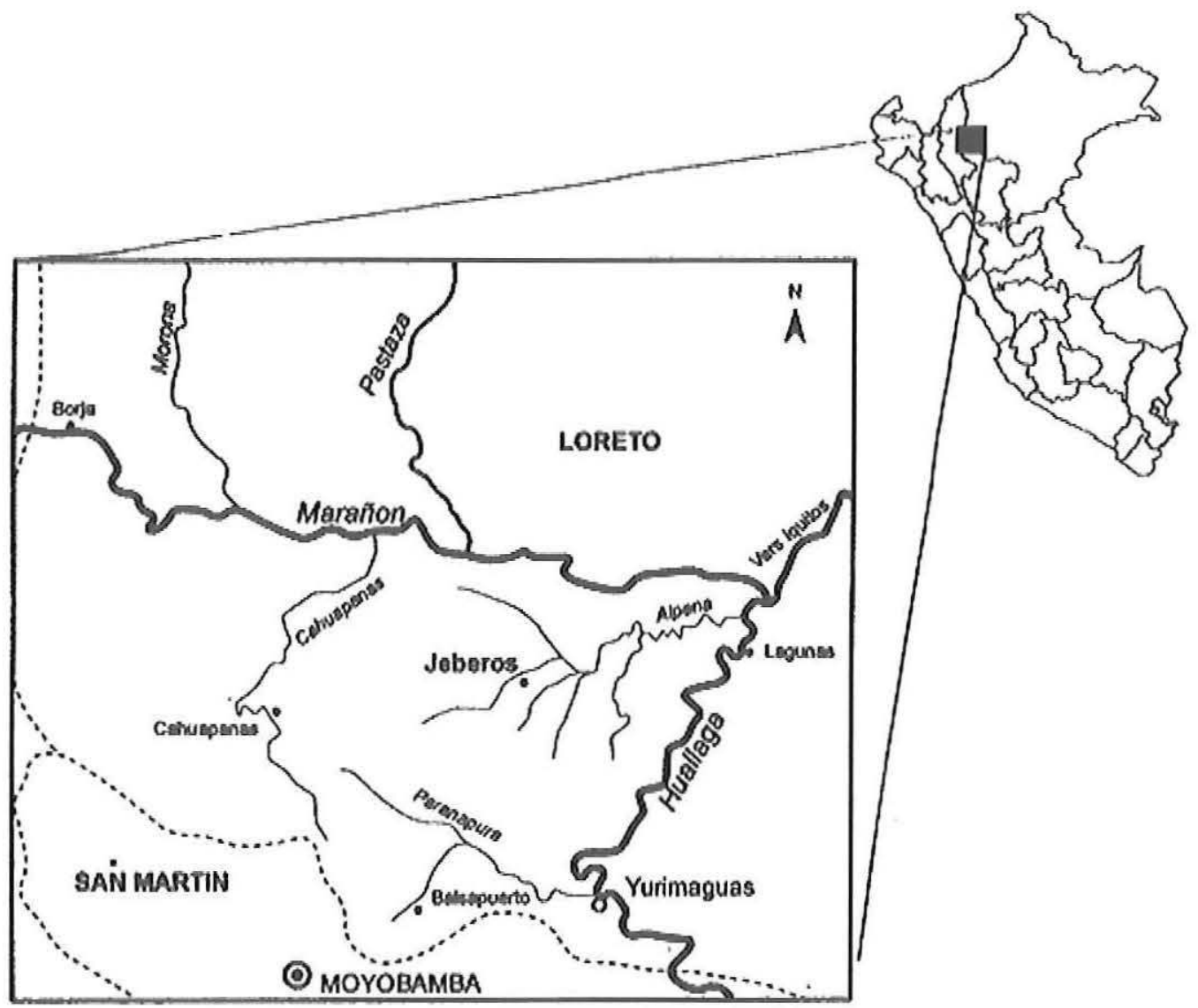

Umilus deperternentetos

(0) Ceptess deporiamentes

- Ceplialo provindalo

- Copitula de diatra

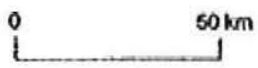

Fig. 1 - Localisation de Jeberos.

de parenté fondée sur la confiance mutuelle et le partage. Souvenir d'une société paisible, respectueuse de ses traditions, qui contrasterait avec l'évolution récente de leur village caractérisé, à les entendre, par le soupçon et la violence fratricide.

Mes hôtes ne se limitaient pas à ce constat amer. Ils en donnaient également une explication : une telle détérioration de leurs relations sociales serait, selon eux, intimement liée à leur adoption récente de modes de comportement estimés 
autrefois propres à leurs voisins indigènes et métis. Des voisins avec qui les Jebero ont justement multiplié les mariages au cours de ces dernières décennies. Or, la vie en co-résidence avec ces représentants de l'altérité, autrefois maintenus à l'extérieur du village, aurait induit un " mimétisme » comportemental aux conséquences délétères : « Avant à Jeberos, on ne tuait pas ainsi. On n'accusait pas non plus comme ça. Les Chayahuita le font et certains à Jeberos ont commencé à les imiter. Mais, avant, ce n'était pas ainsi » (Hermenegildo Mozombite).

Sur ce point encore, les Jebero s'opposent implicitement à leurs ancêtres : le mythe de fondation de la société jebero, sur lequel nous reviendrons, raconte comment celle-ci se serait progressivement constituée au travers des unions incestueuses entre frères et sœurs sur plusieurs générations. Un inceste originel garant d'une autarcie sociale absolue, qui aurait longtemps permis à leurs ancêtres d'échapper aux maux de l'extérieur et d'éviter, à l'inverse d'aujourd'hui, la « contamination » de leurs pratiques relationnelles.

Ce tableau radicalement bipolaire de leur évolution (société harmonieuse/ société divisée ; société incestueuse/société métissée) se révèle en décalage avec la réalité historique du parcours des Jebero et de leur village au cours des derniers siècles. Jeberos reste aujourd'hui particulièrement isolé au sein de la province Alto Amazonas (région Loreto). Situé loin à l'intérieur des terres, ce village de près de deux milles habitants se trouve de fait à l'écart des circuits commerciaux qui animent la région. Cet isolement géographique reflète mal son histoire, en vérité très ancienne. Dès 1640, un prêtre nommé Lucas de la Cueva rassembla les membres du groupe Jebero, fondant ainsi la toute première mission jésuite de l'ancienne province de Maynas. Peuple guerrier récemment allié aux conquérants espagnols, les Jebero devinrent dès lors, et durant toute l'époque coloniale, la figure de proue de l'indianité chrétienne, célèbres pour leur participation active aux milices indiennes chargées d'escorter les missionnaires dans les expéditions de conversion (et de capture) des Indiens « infidèles ». Ces mêmes infidèles étaient souvent amenés de force à la mission pour y être formés. Malgré la présence majoritaire des Jebero, cette mission se présenta donc très tôt comme foncièrement multiethnique. L'organisation sociale jebero garde d'ailleurs encore les traces de cette phase de métissage très ancienne, notamment dans l'opposition binaire entre les deux moitiés (haute et basse) du village. Durant plus de deux siècles, le christianisme s'aflirma comme un dénominateur commun décisif, à la fois garant de la cohésion sociale et marqueur identitaire face à l'extérieur.

Cette indianité chrétienne modèle a été cependant remise en question au cours $\mathrm{du} \mathrm{xx}^{\mathrm{e}}$ siècle avec l'intégration du village jebero aux différents cycles de l'économie régionale et l'impact des importants flux migratoires qui en ont découlé. Les déportations massives vers le Brésil des caucheros, l'implantation de grandes haciendas au sein même du territoire jebero puis, surtout, l'immigration d'une main d'œuvre issue des départements alentours venue exploiter le barbasco (Lonchocarpus nicou) dans les années 1940-1960, ouvrirent la voie à un nouveau 
processus de métissage, effaçant en quelques décennies tout signe extérieur de l'origine amérindienne des habitants du village ${ }^{2}$.

Si, comme je l'ai montré ailleurs (Julou 2006), le rôle de modèle qui leur a été dévolu durant l'époque coloniale avait paradoxalement favorisé la construction d'une singularité « jebero ", le $\mathrm{xx}^{\mathrm{e}}$ siècle les a amenés à s'inscrire, de fait, dans la catégorie de la paysannerie métisse amazonienne. À ce titre, les Jebero peuvent être considérés comme un nouvel exemple de ces «Indiens invisibles », une dénomination adoptée par Stocks (1981) pour les Cocamilla, voisins des Jebero et décrivant assez bien l'ambiguîté du statut de ces populations, certes d'origine amérindienne, mais dénuées de tout signe visible attestant d'un quelconque particularisme identitaire. Une période d'« invisibilité » à laquelle les Jebero ont cependant choisi récemment de mettre fin à travers la création d'une fédération indigène (la Fédération des communautés natives de Jeberos, FECONAJE), chargée de faire reconnaître par l'État péruvien l'identité amérindienne des habitants de ce village. Mon séjour à Jeberos à la fin des années 1990 correspondait donc à une période charnière pour ce peuple en proie à des tensions internes éminemment palpables.

À la lecture de cette brève histoire jebero, il serait tentant d'identifier la détérioration des relations entre les membres du village à des tensions « interethniques » et d'interpréter la création de la Fédération comme la réaction épidermique d'une identité menacée. Dans ma thèse consacrée à ce phénomène d'ethnogenèse (Julou 2006), j'ai montré que ce processus récent de revendication identitaire, loin d'être un sursaut tardif d'un ethos jebero hostile à toute assimilation de l'" Autre », en constituait au contraire le produit direct. L'incorporation généralisée des caractéristiques de l'altérité avait induit une transformation profonde du rapport traditionnel des Jebero aux notions d'identité, de mémoire et de "culture", changement qui conditionnait la possibilité même d'une revendication identitaire face à l'Etat.

Dans le présent article, je souhaite aborder cette question de l'incorporation de l'altérité sous l'angle des relations matrimoniales, si essentielles à la construction du socius amazonien. Je propose notamment de démontrer que les tensions décrites plus haut ne sont pas liées à l'identité des alliés (ces tensions concernaient tout autant des Jebero « de souche »), mais à l'évolution récente de leurs relations mutuelles. Une évolution qui renvoie en réalité à une transformation sensible de leur conception même de l'affinité. Comme nous le verrons, celle-ci se construit habituellement chez les Jebero au travers d'un équilibre toujours renouvelé entre une alliance au sein de sa propre moitié, progressivement assimilée à la consanguinité, et une alliance avec un membre de la moitié adverse, incarnation d'une forme d'altérité, certes menaçante, mais en définitive maîtrisée ${ }^{3}$. Or au cours du $\mathrm{xx}^{\mathrm{e}}$ siècle, un tel équilibre dualiste s'est trouvé mis à l'épreuve par de très nombreux mariages avec des personnes extérieures au village. Destinée à rester inscrite dans une opposition binaire et auto-reproductive, l'aftinité s'est vue soudaine- 
ment et massivement incarnée par des représentants de la société nationale ou des sociétés indigènes voisines. Pour comprendre l'impact réel d'un tel phénomène, j'ai choisi de confronter les figures symboliques de l'aftinité transmises par la tradition orale à l'évolution récente des relations matrimoniales jebero. Comme nous allons le voir, les récits mythologiques proposés ici, une fois mis en perspective avec la réalité actuelle des relations entre alliés, montrent l'émergence d'une figure paradoxale de l'affinité car logiquement destinée à rester extérieure et pourtant désormais installée au cœur de la socialité jebero : parent par alliance, co-résident, l'affin demeure néanmoins un «étranger » dont la proximité accentue le caractère menaçant.

\section{LE FRÈRE OU L'AFFINITÉ INVISIBLE}

Avant de commencer l'analyse du concept d'«affinité » chez les Jebero, revenons sur le mythe de fondation de leur société cité en introduction. Je me limiterai ici à en résumer la première partie ${ }^{4}$ qui pose d'emblée, et de manière particulièrement limpide, les principes structurant cette notion.

Ce mythe raconte l'histoire d'un homme marié à une femme jivaro et vivant dans la communauté de ses beaux-parents. Alors qu'il parcourt son territoire de chasse, il entend les cris de sa fille enlevée par un esprit de la forêt. Parvenant à la récupérer, il apprend de la bouche de son enfant l'infidélité récurrente de sa femme. Toute à ses ébats amoureux, celle-ci ne s'est même pas aperçue de l'enlèvement de sa fille. Revenu seul à sa demeure et mesurant les mensonges et dénégations répétées de sa femme, il finit par la tuer. Puis il prend la fuite avec sa fille afin d'échapper aux représailles de ses beaux-frères jivaro. Sa fuite devient bientôt une quête : celle d'un territoire isolé du reste du monde pour fonder un village échappant à la menace de ses anciens alliés. Afin de peupler ce nouveau village, il entretient des relations incestueuses avec sa propre fille. Un inceste qui se poursuit aux générations suivantes puisque les frères épousent leurs sœurs. Une fois le village constitué, le père fondateur édicte de nouvelles règles de vie censées contraster avec celles de ses anciens beaux-parents jivaro : respect, confiance, générosité, entraide mutuelle. Autant de " lois » qui devront être garantes d'un mode de vie autarcique, à l'abri de la vengeance des Jivaro et, plus généralement, de la violence et du mensonge pratiqués dans le monde extérieur.

Comme l'a bien montré Lévi-Strauss (1962, p. 305), un récit étiologique est surtout démarcatif. L'isolement absolu recherché par le héros et les règles de vie qu'il instaure se veulent, avant toute chose, fondateurs d'une différence explicitement placée en contraste avec l'altérité jivaro. Dans le même temps, et c'est sur ce point que j'insisterai ici, ce mythe exprime également une négation radicale de la notion même d'« affininité ». Au sein du village des ancêtres, l'époux est un frère, l'allié un consanguin. L'affinité, en tant que telle, n'existe pas. Plus précisément, 
elle n'existe plus. Car, au début du récit, celle-ci s'avère au contraire omniprésente : le héros lui-même constitue un allié vivant chez ses beaux-parents jivaro. Une vie marquée par le mensonge, la trahison et le meurtre. Ce n'est que dans un deuxième temps que le héros parvient à construire une nouvelle société délestée de toute affinité et dès lors caractérisée par la confiance et le partage.

D'une certaine manière, ce mythe de fondation du peuple jebero exprime une vision de la parenté qui se rapproche de l'analyse proposée il y a quelques années par Viveiros de Castro (2001, p. 26) : l'affinité est bien une donnée de départ ; la consanguinité, un idéal qui se construit. Leur différence tient dans le fait que, dans le mythe, cet idéal est réalisé. La société jebero se serait construite sur une consanguinité absolue. Par là même, ce récit renvoie l'image d'une société des ancêtres parfaitement close sur elle-même. L'inceste constitue la condition sine qua non de la survie de la société jebero, lui permettant d'échapper aux dangers que représente le monde extérieur. Or, à travers l'image d'une société jivaro vengeresse, cet extérieur menaçant prend précisément la forme symbolique de l'aftinité. Les affins jivaro, dont la menace pèse sur la société des ancêtres comme une épée de Damoclès, représentent ce que Viveiros de Castro (2001, p. 22) a défini comme l'«affinité potentielle» : une affinité symbolique, parfaitement distincte des affins réels, qui sert d'opérateur logique à la construction d'une identité de référence. Cette lointaine affinité jivaro, incarnation ultime d'une forme d'altérité prédatrice, définit par contraste les contours d'une société ancestrale jebero idéalement autarcique.

De fait, dans ce contexte mythique, l'affīnité est explicitement placée hors champ, rendue parfaitement incompatible avec une autre notion essentielle de la parenté jebero : la vie en co-résidence. Les habitants du village jebero sont, à l'aube des temps, tous frères et sœurs. L'affinité, pour sa part, reste tout entière définie et incarnée par les ennemis de l'extérieur. Une opposition simple et radicale dans laquelle, cependant, les Jebero d'aujourd'hui ne se reconnaissent pas : leur village ne leur apparaît plus comme une citadelle assiégée. L'autarcie des temps anciens, le refus obstiné de toute forme d'alliance avec l'extérieur sont dépassés, rendus caduques par leur histoire récente : l'exil d'une part importante de la population villageoise vers le Brésil à l'époque du caoutchouc, puis l'immigration d'une population métisse originaire des départements de San Martin ou de Loreto durant les années de gloire du barbasco ont définitivement mis fin à l'idéal de consanguinité.

Un idéal souvent, d'ailleurs, pris au pied de la lettre par les Jebero et dont ils se démarquent vigoureusement : lorsque j'étais amené à faire référence à ces anciennes unions entre " frères » et « sœurs » mentionnées par leur mythe d'origine, ils m'indiquaient avec force l'interdiction qui leur était faite de s'unir avec tout consanguin direct, y compris donc leurs cousins germains. À l'instar de leurs voisins chayahuita, les Jebero étendent la notion de germanité aux cousins bilatéraux du premier degré. La terminologie vernaculaire des liens de parenté 
est, à la génération d'Ego, de type hawaïen et ne prend donc pas en compte la distinction entre cousins croisés et cousins parallèles, considérés ici comme des frères et sœurs classificatoires. Par ailleurs, les Jebero ne s'estiment aucunement limités dans le choix de leur conjoint. L'absence de toute prescription matrimoniale conjuguée à l'extension de la notion d'inceste aux cousins germains semblerait dès lors ouvrir un champ presque illimité des possibilités d'union, justifiant ainsi le contraste ressenti par les Jebero face à la consanguinité soigneusement entretenue par leurs ancêtres. Comme nous allons le voir pourtant, la représentation mythique de l'époux-frère conserve sa dimension performative dans les stratégies matrimoniales jebero.

Un premier recensement (Figure 2) m'amena tout d'abord à distinguer trois catégories d'unions: les mariages au sein de sa propre moitié, les mariages inter-moitiés et les mariages avec des personnes extérieures au village ${ }^{5}$. Mon enquête statistique mettait l'accent sur une tendance endogamique des moitiés. Plutôt qu'une règle explicite imposant le mariage au sein de sa moitié, les Jebero préféraient y voir l'expression d'une préférence empirique : « on aime se marier près de notre famille ». Cette préférence affichée envers un conjoint " proche» doit tout d'abord s'interpréter dans sa dimension proprement spatiale. Comme le laissait deviner leur mythe fondateur, le mariage jebero est traditionnellement uxorilocal et implique donc pour l'époux de se déplacer, de quitter ses consanguins pour partager la vie de ses affins. Or épouser une femme voisine, membre de sa propre moitié, permet de limiter fortement la portée effective de ce déplacement. Si l'homme rejoint de fait la maison de son beau-père, il restera souvent à proximité immédiate de ses parents et sera en mesure de maintenir avec eux des liens réguliers, poursuivant ainsi sous certains aspects (visites, entraides) une co-résidence avec ses consanguins.

\begin{tabular}{|c|c|c|c|}
\hline MARIAGKS & $\begin{array}{l}\text { INTEIRNES } \\
\lambda \text { I I A MOITIE }\end{array}$ & INTERMOITIES & A L'EXTEGRIEUR \\
\hline $\begin{array}{c}\text { ACTUEIS } \\
\text { (Depuis les années 1970) }\end{array}$ & $63,6 \%$ & $13,6 \%$ & $22,8 \%$ \\
\hline $\begin{array}{c}\text { ANTERIEURS } \\
\text { (période du barbasco et } \\
\text { hacienda) }\end{array}$ & $51,6 \%$ & $10,1 \%$ & $38,3 \%$ \\
\hline MOYENNE & $56,6 \%$ & $11,6 \%$ & $31,8 \%$ \\
\hline
\end{tabular}

FIG. 2 - Statistique des unions matrimoniales jebero.

Devant mes questions quelque peu artificielles, les Jebero aimaient indiquer : " mes parents sont les gens avec qui je vis tous les jours ». Ils faisaient ici référence à ce qu'ils appellent communément les parents proches ou « vrais ». Un critère de 
" vérité » lié, à les entendre, à la confiance qu'ils peuvent leur accorder. Or cette confiance se mesure essentiellement à l'aune des expériences communes vécues avec une personne. Les expéditions de chasse ou de pêche, l'essartage et l'entretien des jardins, le partage des nourritures et boissons sont des activités qui réunissent régulièrement les membres d'une même famille. La notion de parents proches renvoie donc avant toute chose à l'ensemble des personnes avec qui on partage les activités nécessaires à sa propre subsistance.

Cependant, se marier avec une personne " proche " possède également une autre consonance dans l'esprit des Jebero. Cette notion de proximité fait implicitement écho à l'existence de liens antérieurs entre les deux familles amenées à s'allier à travers une union. Liens nés d'une certaine forme de co-résidence au sein de laquelle les deux familles auront souvent eu l'occasion de s'entraider, de partager nourritures et boissons; liens surtout issus d'alliances conclues à des générations antérieures ou à la même génération que le couple par leurs parents collatéraux respectifs. De fait, la fréquence des mariages au sein d'une même moitié amène chaque famille à se trouver inscrite dans différents réseaux d'alliances régulièrement entretenus. Une part importante des nouvelles unions se limite donc souvent à renforcer une alliance déjà éprouvée entre deux groupes de parentèle.

À cet égard, la famille de Rozendo Inuma Iumbato et Alicia Huiñapi Lomas servait souvent de référence. " Eux ont réussi », me résumait ainsi Fidel Lomas, membre de la moitié basse. Intrigué par ce commentaire admiratif, je rendis un jour visite à Rozendo dont la demeure était située à la pointe de la moitié haute. Les choix matrimoniaux observés au sein de sa famille me permirent de constater de manière empirique ce que les Jebero considéraient comme l'alliance idéale (Figure 3).

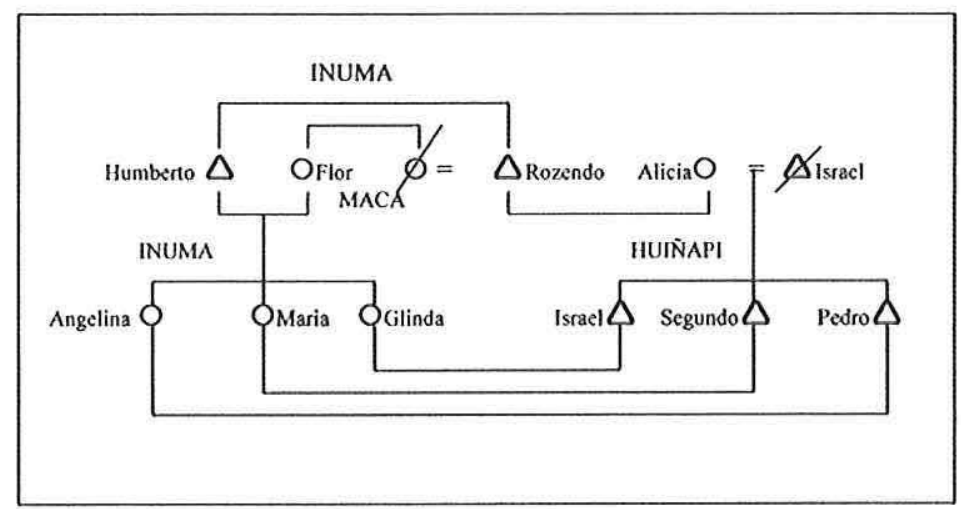

FIG. 3 
Rozendo et Alicia, tous deux veufs d'un premier mariage, avaient choisi il y a quelques années d'unir leurs destinées. Cela n'aurait bien sûr rien d'exceptionnel si ce n'était qu'auparavant les trois nièces de Rozendo (les filles de son frère Humberto) avaient elles-mêmes épousé les trois fils d'Alicia.

Qu'avaient-ils réussi précisément ? Si l'on se réfère à la préférence affichée par les Jebero, les enfants d'Humberto et d'Alicia s'étaient effectivement mariés à proximité immédiate de leur propre famille : membres de la même moitié, ils se connaissaient depuis toujours. Cependant, en se mariant ainsi entre beaux-frères, ils avaient surtout respecté un principe clé des stratégies matrimoniales jebero, à savoir limiter la diversité des alliances : les frères Huiñapi avaient tous, en effet, intégré la même famille alliée.

Percevoir ces choix matrimoniaux comme relevant d'une stratégie consciente n'est pas faire outrance à la réalité du terrain. Certains, comme Fidel Lomas, l'admettaient de manière tout à fait claire : " la coutume est de se marier avec une seule famille. Lorsque le premier frère épouse une jeune fille, ses frères cadets essaient d'épouser les sœurs de celle-ci pour qu'il n'y ait pas trop de familles à unir. Ainsi on évite les problèmes ».

Les mariages Jebero doivent être abordés selon une double perspective. L'une personnelle, consistant à choisir un conjoint « proche » afin d'éviter les désagréments d'une alliance avec une famille méconnue. L'autre, plus collective, nécessite d'accorder son propre choix à ceux de ses parents collatéraux. Cette recherche de proximité serait peine perdue si, en parallèle, ses propres consanguins optaient pour des alliances divergentes. En l'absence d'une certaine harmonisation des alliances, la multiplication des affins augmente d'autant les risques de tensions et de conflits. Or seul le redoublement des alliances unissant à différents niveaux deux familles proches permet de limiter au maximum la diversité effective des relations d'affinité découlant de ces unions.

Pourtant, ce mariage entre beaux-frères ne suffit pas à expliquer la dimension paradigmatique de l'alliance des enfants d'Humberto et d'Alicia. Nous verrons que ce type de mariage et sa variante, l'échange sororal, sont monnaie courante dans le village jebero ${ }^{6}$. La réussite de cette alliance réside dans le mariage de Rozendo et d'Alicia. Leur union ne se limite pas à assurer l'hermétisme de cette succession d'alliances. Alicia a choisi de s'unir à l'oncle de ses propres belles-filles. Ces dernières ont donc désormais pour beau-père un parent consanguin. Leurs propres mariages avec les frères Huiñapi prennent dès lors l'apparence de la consanguinité.

Un tel processus trouve écho dans un aspect de la terminologie de parenté jebero. Ego féminin nommerait son beau-père par un terme censé désigner le frère de son père, papashugsha. La belle-mère serait, quant à elle, désignée par le terme ashatuksha, soit « sœur de mère ». Prise au pied de la lettre, cette terminologie impliquerait deux conséquences logiques: tout d'abord, à la génération des parents d'Ego, le frère de son père aurait épousé la sœur de sa mère. Ce mariage 
entre beaux-frères est en soi chose possible. Comme nous l'avons vu, il apparaît même comme l'union " préférentielle » pour les Jebero. La terminologie vernaculaire se limiterait donc ici à exprimer une pratique attestée dans la société. À l'inverse, la seconde implication des équivalences HF:FB; HM:MZ apparaît au premier abord plus incongrue : Ego aurait épousé le fils de son oncle paternel et de sa tante maternelle, soit un cousin « doublement » parallèle considéré par cette même terminologie comme un « frère ». Or ce type de mariage ne correspond à aucune réalité de fait dans la société jebero. Cette particularité terminologique s'inscrit dans une logique d'effacement de l'aflinité : en désignant ses beauxparents par des termes servant habituellement à désigner les germains bilatéraux de ses propres parents, la femme " stipule » la consanguinité de l'alliance instaurée avec la famille de son époux ${ }^{7}$. L'origine extérieure de ce dernier est ainsi oblitérée au profit d'une affirmation, tout au moins nominative, de l'existence de liens de consanguinité avec la famille de sa femme, chez qui il est amené à vivre en application du principe d'uxorilocalité. De fait, si les parents de l'époux sont désignés comme frère de père et sœur de mère, l'époux lui-même est implicitement placé dans une position de "frère » vis-à-vis de sa propre femme.

Or telle est précisément la conclusion que nous pouvons émettre au sujet des alliances entre les familles Inuma et Huiñapi. À travers le mariage de Rozendo et Alicia, ce qui n'est habituellement que stipulation nominative devient réalité empirique. Rozendo incarne les deux dimensions du papashugsha, à la fois oncle et beau-père de Glinda, Maria et Angelina. Dès lors, leurs époux, ayant pour " père » l'oncle de leurs femmes, occupent, sur un mode classificatoire, la position de cousins germains, soit de « frères ». À l'origine sans aucun lien consanguin avéré, ces deux générations d'alliés sont parvenues à « former une seule famille », selon les termes mêmes de Fidel. Un tour de force justifiant l'admiration des habitants du village : le mariage d'Alicia et Rozendo permettait d'unir si étroitement leurs deux familles que le principe même de l'affinité tendait à s'effacer au profit d'une communauté élargie de parents placés désormais sous le signe de la consanguinité. L'affin, dans un tel contexte, a perdu toute épaisseur. Il est devenu invisible.

Cette analyse nous permet également de percevoir sous une autre lumière le mythe d'origine jebero. On ne peut manquer d'être frappé par la correspondance entre cet aspect de la terminologie, qui identifie l'époux à un " frère », et l'affirrmation posée dans le mythe selon laquelle l'inceste généralisé entre « frères » et "sœurs " aurait donné naissance à la société des ancêtres. Appréhendé de manière littérale, ce récit évoque des pratiques aberrantes dont se démarquent vigoureusement les Jebero. Inscrit dans un processus social et terminologique où la consanguinité de l'affīn sert de postulat nécessaire à sa bonne intégration, la référence ancestrale à des relations matrimoniales entre germains retrouve tout d'un coup toute sa pertinence dans la société jebero actuelle.

Observons à présent différents modes d'alliance pratiqués dans le village qui tentent de s'approcher au mieux de l'idéal atteint par la famille de Rozendo et 
d'Alicia. Toutes générations confondues, les mariages entre beaux-frères, incluant l'échange sororal, représentent environ $20 \%$ des pratiques recensées. Si ce type d'union n'est pas aujourd'hui statistiquement majoritaire, il constitue toujours, on l'a vu, l'alliance préférentielle pour les Jebero. Une telle stratégie est néanmoins très diffícile à réaliser dans les faits. Elle souffre en effet d'impondérables, notamment démographiques, qui la rendent souvent inapplicable : la différence d'âge entre beaux-frères, l'absence de symétrie entre les nombres respectifs de germains au sein des familles alliées ou la mort prématurée de l'un d'entre eux empêchent régulièrement l'harmonisation des alliances entre deux groupes de parentèle.

Ces limites intrinsèques à tout échange matrimonial de type "restreint " suscitent des adaptations de la part des Jebero qui cherchent par des voies détournées à reproduire le même schéma.

Nous retrouvons dans la Figure 4 la même situation de départ que celle des trois fils d'Alicia : trois frères cherchent à harmoniser leurs alliances respectives. Ils n'ont pu épouser trois sœurs, mais leurs mariages démontrent une volonté consciente de trouver conjoint au sein d'une même famille. Ainsi, Francisco, bien plus jeune que son frère Roldan, n'a pu épouser sa belle-sœur, mais la fille de celle-ci. Dans la perspective du mariage préférentiel entre beaux-frères, Maria devient un substitut adéquat à sa mère, Nelsa. Robinson a, quant à lui, choisi d'épouser Irma, la fille de la cousine parallèle (soit la « søur ») de sa belle-søur. Les trois frères ont épousé trois femmes qui ont entre elles des rapports étroits de consanguinité.

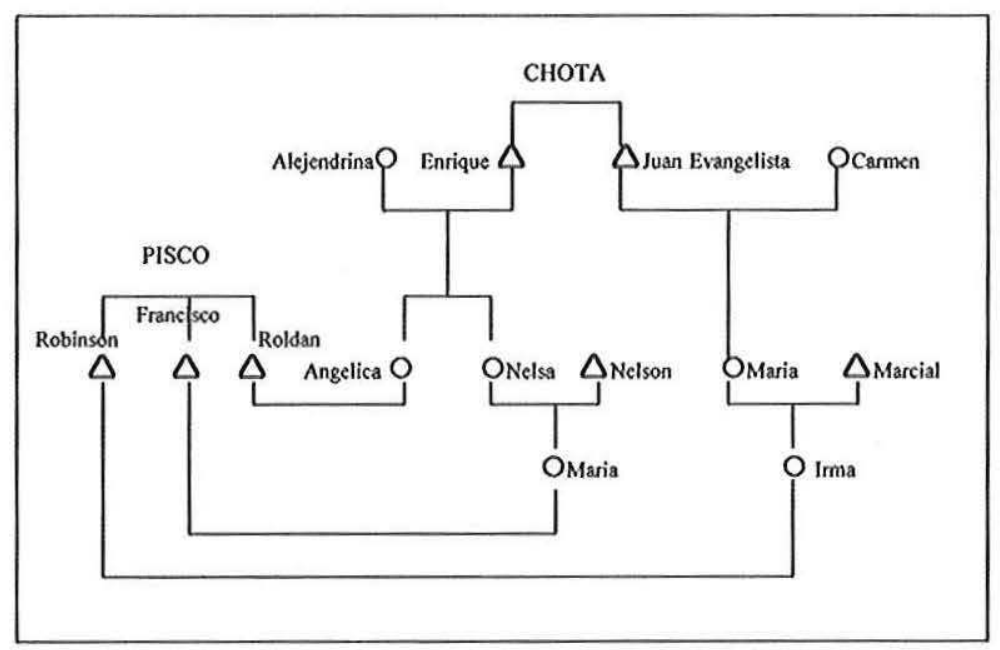

FIG. 4 
Précisons maintenant : Enrique Chota, qui a épousé une femme non jebero, et son frère Juan Evangelista, lui-même marié à une femme de la moitié haute, sont restés vivre sur les terres de leur enfance, au sein de la moitié basse ${ }^{8}$. De ce fait, Irma, Maria et Angelica ont toujours vécu à immédiate proximité les unes des autres. Les frères Pisco se sont donc mariés au sein d'une seule famille concentrée dans un même espace. Ils ont effectué le même déplacement, leur permettant de poursuivre en commun leurs activités de chasse.

L'échange sororal, s'il ne permet pas en principe ce maintien de la co-résidence entre frères, favorise un resserrement des liens unissant deux familles alliées. Les exemples suivants montrent que, lorsque l'échange direct ne peut être assuré, celui-ci peut se trouver, comme le mariage entre cuñados, différé à la génération suivante (Figure 5).

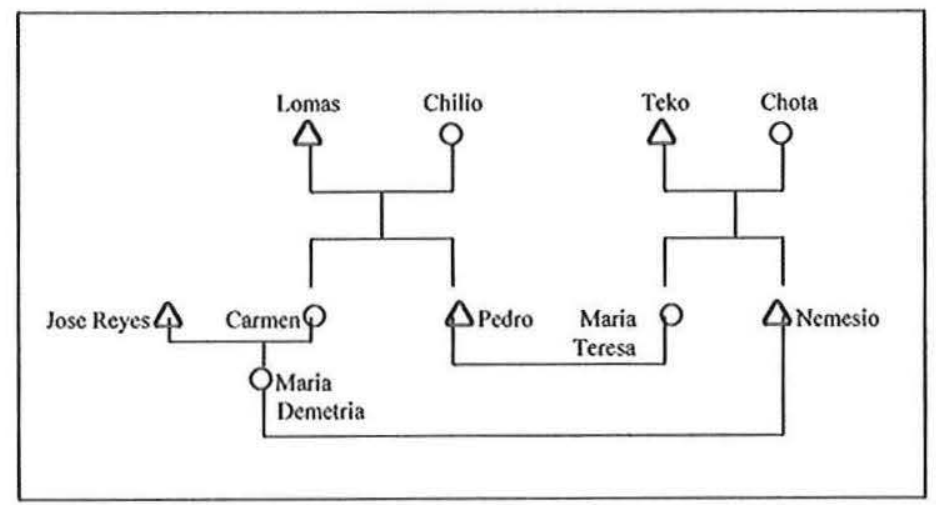

FIG. 5

L'extension de la notion de germanité à l'ensemble des cousins du premier degré autorise également d'autres variantes comme le montre la Figure 6.

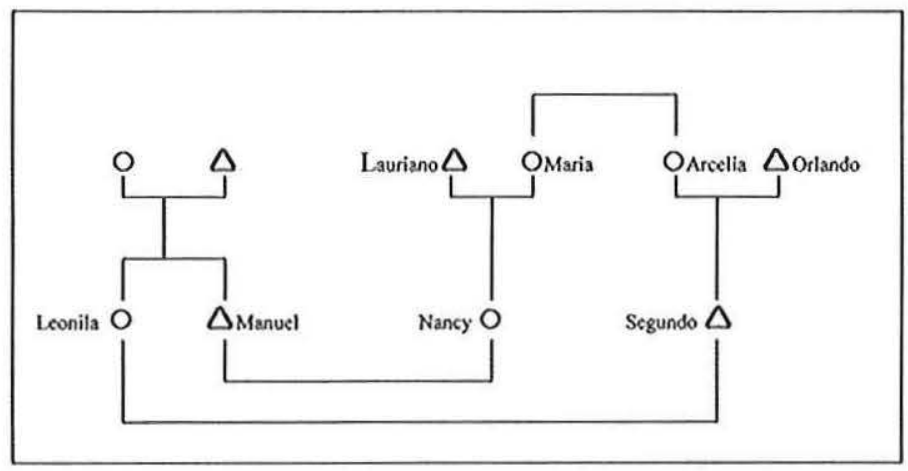

FIG. 6 
Arcelia et Maria, respectivement mères de Segundo et Nancy, sont sœurs et vivaient, suite à leurs mariages avec des membres de la moitié haute, à proximité immédiate l'une de l'autre. Segundo et Nancy, cousins parallèles et co-résidents, se considèrent donc comme " frère » et « sœur ». Or, en se mariant avec Leonila et Manuel eux-mêmes frère et sœur biologiques, ils ont réalisé une forme alternative d'échange de sœurs.

La Figure 7 permet de visualiser sur trois générations ces mariages préférentiels entre beaux-frères et leurs variantes.

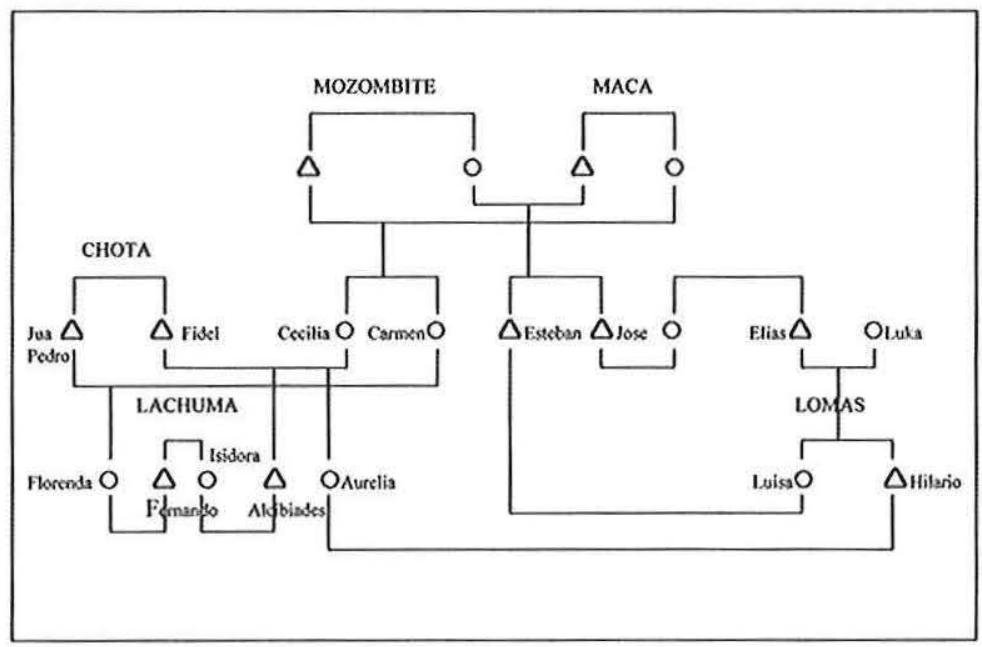

FIG. 7

À la génération supérieure, les familles Maca et Mozombite ont pratiqué l'échange sororal direct. Les deux filles Mozombite Maca, Cécilia et Carmen, ont épousé deux frères, Juan Pedro et Fidel, tandis que les deux fils Maca Mozombite (Esteban et José) ont pratiqué cette variante différée du mariage entre beauxfrères déjà observée dans la Figure 4. Florenda et Alcibiades étaient des cousins doublement parallèles, soit "frère » et "sœur » co-résidents. À l'instar de Segundo et Nancy (Figure 6), leurs conjoints étaient entre eux frère et sœur. Leur double union équivaut donc également à un échange de sœur sur un mode classificatoire. Enfin, l'union d'Hilario et Aurelia constitue, en dépit des apparences, une simple variante du mariage entre beaux-frères. Esteban constitue le personnage central permettant de justifier cette interprétation. Il est à la fois le cousin croisé de la mère d'Aurelia, soit le "frère " de sa mère, et le beau-frère de la tante d'Hilario. Il est devenu par la suite le beau-frère de ce dernier en épousant Luisa. Hilario et Esteban se reconnaissent mutuellement comme cuñados. Or, dans la perspective d'une conformité au mariage préférentiel 
entre beaux-frères, Hilario aurait dû chercher à épouser une sœur d'Esteban. Les circonstances ont rendu cet objectif impossible : Esteban n'a pas de sœur et ses cousines croisées, plus âgées, étaient déjà mariées. Hilario a dès lors jeté son dévolu sur la fille de la "sœur" de son beau-frère : nous retrouvons là une nouvelle variante de la Figure 4.

Cette union serait également susceptible d'une autre interprétation. Il est intéressant de noter que les deux époux étaient considérés comme des " cousins éloignés » (primos lejanos en espagnol) avant leur mariage, ceci bien qu'il n'existait entre eux aucun lien avéré de consanguinité. Leur exemple nous confronte au problème d'interprétation posé par l'utilisation de la terminologie espagnole et notamment par l'ambiguïté de la notion de cousin. Les Jebero distinguent deux catégories de cousins. L'expression " cousins germains » (primos hermanos en espagnol) constitue la traduction la plus adéquate aux termes vernaculaires yugsha et kaigsha (pour un ego féminin), yalig et shagsha (pour un ego masculin), "frères et sœurs ", soit une catégorie de parents avec lesquels le mariage est prohibé. À l'inverse, l'expression " cousins éloignés » renvoie à une réalité beaucoup plus floue. Si elle désigne prioritairement des cousins au deuxième degré, elle servira également de terme d'adresse envers une personne membre du village appartenant à la même catégorie d'âge que le locuteur, mais avec qui n'existe aucun lien de parenté ou d'affïnité. Il s'agit en l'occurrence de termes de politesse ou d'affection utilisés en présence de l'intéressé.

Parfois cependant, cette expression renvoie à des liens de parenté indirects, mais bien réels. Dans le cas d'Hilario et d'Aurelia, c'est José, mari de la tante paternelle d'Hilario qui joue ici le rôle clé. En effet, par son union avec sa tante, José est dénommé en espagnol tío, soit l'équivalent terminologique de « frère de mère ou de père ». Or cet " oncle » a pour " sœur » la mère de son épouse. En appliquant à la lettre la terminologie de parenté découlant de cette alliance, nous obtenons la Figure 8.

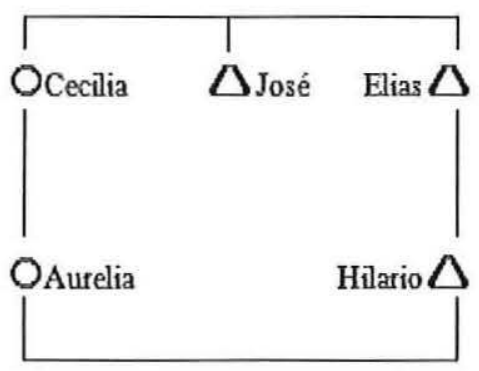

FIG. 8

La succession d'alliances observée dans la Figure 7 a conduit Hilario et Aurelia à s'inscrire dans une position de germanité classificatoire, justifiant ainsi 
leur référence mutuelle comme "cousins éloignés ». Leur union peut dès lors apparaître comme le moyen de renforcer la cohésion de cette nouvelle communauté de parents. Elle nous permet également d'entrevoir l'attitude inverse, mais symétrique, adoptée face aux deux catégories de cousins. Si l'union des cousins germains s'apparente à un inceste entre frère et sœur réels et se trouve en conséquence rigoureusement condamnée, le mariage entre cousins éloignés, perçus ici comme des frères et sœurs " fictifs », participe de ce processus de " consanguinisation » des familles alliées. Rejetée dans le cadre de la famille étendue des cousins germains, la consanguinité est reconstruite à un niveau supérieur. De fait, si la logique hawaïenne impose une ouverture plus large des relations d'aftĩnités virtuelles, le redoublement des alliances assure la concrétisation de l'idéal jebero : garantir la convivialité harmonieuse des phratries.

À cet égard, les Jebero expriment une logique très généralisée en Amazonie. La valorisation extrême des principes de co-résidence et de convivialité dans la définition d'un ensemble de " parents », les stratégies matrimoniales de redoublement, la dimension performative et non prescriptive de la parenté jebero constituent en réalité des traits récurrents chez nombre de peuples amérindiens de la région (voir notamment Overing 1975; Gow 1991 ; Viveiros de Castro 2002 ; Vilaça 2002a). Tout juste peut-on avancer l'hypothèse que cette logique se révèle particulièrement accentuée dans le cas des peuples marqués, comme les Jebero, par leur histoire au sein des missions. Vilaça (2002b) a ainsi montré comment la conversion des Wari' au chritianisme a contribué à renforcer le processus traditionnel de consanguinisation des alliés, rejetant l'affinité en dehors du groupe local. Selon cet auteur, « l'idéal chrétien de conduite avait un rapport avec l'idéal wari' de consanguinité généralisée » (ibid., p. 63).

Cependant, comme je l'ai évoqué en introduction, un tel idéal s'est trouvé confronté chez les Jebero à la réalité d'un processus de métissage particulièrement accentué. Je propose pour la suite d'analyser deux figures mythiques de l'affinité qui permettront de mieux appréhender la manière dont les Jebero conçoivent les modalités et conséquences d'une union placée en dehors du cadre rassurant de la consanguinité.

\section{LA CHAUVE-SOURIS OU L'ALLIÉ SÉDUCTEUR}

Les diverses formes d'unions matrimoniales présentées précédemment répondent au sens commun jebero préconisant les mariages "proches». Ce critère de proximité possède comme pendant tautologique la réticence envers les mariages "lointains ». Ainsi, en opposition à l'idéal de consanguinité aftǐché dans le mythe d'origine, un autre mythe consacré à la relation entre une femme et un homme chauve-souris stigmatise l'union matrimoniale avec des personnes par trop différentes : 
Auparavant, il y avait un petit homme qui n'avait pas de famille. Il vivait seul. Un jour, il rencontra une jeune fille qui vivait avec sa mère. Le petit homme tomba amoureux de cette fille et l'obtint de sa mère. À ce moment-là, la fille ne savait pas qu'il était un vagabond, qu'il n'aimait pas travailler. Durant le jour, il dormait, mais la nuit, il sortait au dehors, transformé en chauve-souris (Desmodus) pour manger des fruits ou sucer le sang des animaux ou des hommes. Il endormait ses victimes en chantant : " ayayaya ». Il ne faisait pas de jardin. Il ne rapportait rien à sa femme. La jeune femme se réveillait, seule, avec la faim et se demandait : « Où est-il ? ». À cinq heures du matin, elle le voyait revenir quand elle-même se levait. Lui allait dormir. Ils ne vivaient pas dans une maison, mais dans le creux d'un gros arbre, le Huaira caspi (?) (tandjilug). Ils dormaient dans le trou.

Lorsque venait une fête, il allait seul danser avec d'autres femmes. Certaines amies de la jeune femme allaient la voir : " Pourquoi ne t'en vas-tu pas ? Dans les fêtes, ton époux est avec d'autres femmes ». On dit qu'il avait beaucoup de femmes.

Un jour, la jeune femme exigea qu'il lui fasse un jardin : « Tu n'apportes rien à manger, tu t'en vas la nuit et tu ne chasses rien. Fais-moi donc un jardin ». Alors, il alla en forêt pour ouvrir un jardin. La belle-mère, ne voyant pas les résultats venir, se rendit sur les lieux, en forêt. Alors, elle le vit, transformé en chauve-souris, volant d'arbre en arbre, se collant au tronc sans pouvoir le faire tomber. La belle-mère se dit : "Comment cet homme se transforme-t-il en animal ? ». Elle revint à la maison et parla avec la jeune fille. Celle-ci lui répondit : « Écoute, je t'avais dit qu'on ne peut unir deux personnes qui ne se connaissent pas. Moi, je ne voulais pas de cet homme, mais tu as insisté et vois ce qui se passe. Mais je ne peux plus le laisser maintenant ».

Durant la nuit, l'homme chauve-souris avait en fait un pouvoir. Il adressait des chants magiques à la jeune femme pour qu'elle ne se comporte pas mal : on pouvait dire n'importe quoi, elle resterait avec lui. Avec ces chants, elle restait endormie et ne savait pas quand il partait. C'était de cette manière qu'il avait tant de femmes : toutes les femmes le voyaient attirant et le désiraient. Il leur adressait des chants magiques. Sa tête était comme un breuvage, son esprit était un breuvage et il lui suffisait de chanter pour envoyer ce breuvage dans l'esprit des femmes.

L'homme chauve-souris réussit finalement à faire son jardin avec l'aide de l'hommevent. Celui-ci souffla très fort pour faire tomber les arbres. La belle-mère vint voir une nouvelle fois et se réjouit de voir cet immense jardin prêt à être brûlé. L'envie lui prit alors de s'emparer d'un morceau d'arbre tacarpa (leliuneg) au milieu du jardin. « Je vais le mettre au milieu du jardin et de là je vais planter ». Pendant ce temps, caché et observant, l'homme chauve-souris commença à chanter pour que la belle-mère redevienne jeune et, au moment où elle se trouvait au milieu, il s'envola et mit le feu tout autour du jardin. La vieille femme brûla, puis aussitôt se remit à vivre. L'homme chauve-souris revint entre-temps à la maison: "Ta mère est morte brûlée dans le jardin ». " Pourquoi est-elle allée brûler le jardin sans prévenir ? ", dit la jeune femme, "Allons là où a brûlé ma mère ». Elle s'en alla avec l'homme jusqu'à l'endroit. Elle, pleurant : "C'est là qu'elle a brûlé ». Mais soudain, elle vit sa mère, rajeunie, joyeuse, travaillant le jardin, occupée à planter. L'homme chauve-souris dit alors : « Regarde comme elle est jeune et travaille bien, mais surtout ne la touche pas ». La jeune femme dit : « Pourquoi n'irais-je pas voir ma mère après avoir tant pleuré, croyant qu'elle était morte ! Suis-je en train de rêver ? ». L'homme chauve-souris : " Non, tu ne rêves pas, 
c'est la réalité ». La belle-mère, jeune, chantait, occupée à semer le manioc. La jeune femme courut pour l'embrasser. L'homme chauve-souris s'exclama : " Ne la touche pas ! », mais elle ne lui prêta pas attention. La belle-mère, au contact de la jeune femme, redevint cendres. Alors, l'homme chauve-souris dit à la jeune femme: «Tu vois, maintenant oui, tu as perdu ta mère. Tu ne m'as pas écouté. Tu ne peux plus la récupérer et le bâton aussi s'est converti en cendres ").

Plus tard, la jeune femme retourna en ce lieu où mourut sa mère pour pleurer. Elle y resta endormie sur un tronc abattu. L'esprit de la belle-mère vint alors la visiter et lui dit : « Pourquoi m'as-tu laissée mourir ? Pour cela tu mourras toi aussi dans quelque temps. Si tu n'avais pas fait cela, personne ne mourrait dans le monde. Tu as ôté la vie à tous ». Elle prit alors ses distances, s'éloigna : « Je ne peux plus manger désormais ces fruits avec toi. Je ne suis plus de cette vie ». Mais la jeune femme l'implora : « Aide-moi depuis là-bas, je n'ai plus ton bâton ». La belle-mère lui répondit : « Non, je ne vais plus t'aider et tu vivras comme tu peux, sinon tu périras ». Ainsi se retira la belle-mère. (Rozenda Maca Lomas)

L'enseignement principal qui pourrait être tiré de ce mythe est explicitement formulé par l'épouse de l'homme chauve-souris : il ne faut pas épouser des inconnus. Or l'homme chauve-souris est présenté, dès le début du récit, comme une personne solitaire, dépourvue de tout parent. Cette situation de départ implique l'absence de liens de parenté préalables entre l'homme et la femme. Le premier est un étranger inscrit en dehors de la sphère des " parents » avec qui la jeune femme et sa mère inaugurent une relation d'affinité. Le mythe nous donne implicitement la raison qui a rendu possible cette union : l'héroïne vit seule avec sa mère, veuve ou séparée de son mari. La famille au sein de laquelle l'homme chauve-souris a choisi sa conjointe semble donc elle-même isolée, sans hommes pour subvenir aux besoins de ces deux femmes, apparemment privées de parents proches chez qui trouver un meilleur époux pour la jeune femme.

La distance qui sépare la femme de son époux est très vite confirmée par le mode de vie pratiqué par cet affin, en parfaite contradiction avec celui de sa jeune épouse : il vit la nuit, ne se nourrit que de fruits et de sang et, à l'inverse d'une société valorisant à l'extrême le principe de sédentarité, s'avère un perpétuel vagabond. Pleinement différent, l'homme chauve-souris possède certaines caractéristiques dont est soupçonné tout affīn préalablement exclu du champ de parenté classificatoire de ses alliés : c'est un séducteur paresseux et doué de pouvoirs magiques. Pour les Jebero, l'homme chauve-souris est le symbole de la séduction abusive, inscrite en dehors du contexte du mariage. Le mythe s'en fait l'écho, qui le présente doué d'un pouvoir lui garantissant l'adhésion des femmes à ses désirs sexuels. Paresseux, poussé par des désirs illégitimes envers d'autres femmes que la sienne, l'homme chauve-souris possède de fait des pouvoirs magiques hors du commun. Un aboutissement logique pour les Jebero : ces derniers établissent un lien étroit de cause à effet entre la paresse, la convoitise et 
la sorcellerie. Trois traits caractéristiques que l'on peut réduire, à la lumière du mythe, à un quatrième terme : celui de l'affinité. L'homme chauve-souris, on l'a $\mathrm{vu}$, est d'entrée de jeu présenté comme un non-parent : il n'en a pas. En ce sens, il est l'« affin » par excellence : son union avec la jeune femme inaugure une relation d'affinité qu'il est impossible, au départ, d'assimiler à une relation entre parents classificatoires. Mais cette assimilation reste par la suite tout aussi impossible. Sa paresse et son vagabondage l'amènent à déroger aux règles implicites de la co-résidence (le partage des activités et des nourritures), indispensables à l'obtention du statut de parent. Cette distance ineffaçable, dans laquelle se maintient l'homme chauve-souris, est corrélative, dans l'esprit des Jebero, des pouvoirs magiques dont il fait preuve.

Remarquons que ces pouvoirs ne sont pas tous foncièrement négatifs. Dans la dernière partie du mythe, il s'avère capable d'offrir l'immortalité à l'humanité toute entière. La relation avec cet affin constituait donc une occasion unique d'échapper à la condition de simple mortel. Cependant, à peine obtenu, ce don disparaît à jamais du fait du refus de la femme d'écouter les avertissements de son mari. À mon sens, cette perte de l'immortalité est moins perçue ici comme un fait regrettable qui pousserait les Jebero à renouveler l'expérience des mariages avec des étrangers que comme une conséquence inhérente à toute relation avec des affins très différents. L'inattention de la femme aux propos de son mari n'est que l'aboutissement d'un phénomène constant dans leurs relations marquées par des rythmes de vie opposés : l'incompréhension mutuelle.

Ces aspects de la relation entre la jeune femme et l'homme chauve-souris se retrouvent dans toute relation d'affinité placée en dehors de tout lien de parenté préalable. Or, si le mythe semble se référer en priorité aux unions matrimoniales avec des non-Jebero, les mariages inter-moitiés en offrent pourtant un exemple immédiat : l'affin originaire de la moitié opposée apparaît aux yeux de la famille de son épouse sous les traits de l'homme chauve-souris.

Comme il a été dit en introduction à cette analyse, tout système dualiste tend à construire en son sein une figure d'altérité représentée par la moitié adverse. L'affin, membre de l'autre moitié, participe donc le plus souvent d'une forme d'extériorité qui peut justifier les analogies que je serai amené à évoquer entre cet affin et certaines métaphores de l'altérité construites dans les mythes. Cette nécessité logique se trouve renforcée à Jeberos par l'endogamie des moitiés. Le taux particulièrement faible d'unions inter-moitiés rend les membres respectifs mutuellement distants en terme de parenté réelle. $\mathrm{Si}$, comme je l'ai montré, la préférence affïchée pour le redoublement des alliances amène à représenter l'affin « idéal » comme une personne dont le vécu commun avec ses consanguins permet son assimilation à un parent, les membres de l'autre moitié, pourtant objectivement proches sur le plan spatial, ne répondent que rarement à cette seconde exigence: les mariages inter-moitiés inaugurent le plus souvent des relations d'affinité entre des familles sans liens antérieurs. 
Au-delà de leur rareté, la particularité des contextes conduisant à ces unions apporte déjà une première confirmation de l'identité implicite posée entre l'homme chauve-souris et l'affin originaire de l'autre moitié : notons tout d'abord qu'au sein des $11,5 \%$ d'unions inter-moitiés, un tiers se rapporte en fait à des unions « illégitimes », c'est-à-dire non officialisées par une vie commune du couple, mais ayant abouti à la naissance d'un ou plusieurs enfants. Cette situation correspond à des relations entre jeunes célibataires qui, en dépit de la naissance d'enfants, n'ont jamais vécu ensemble, le jeune homme finissant par se marier à l'intérieur de sa moitié.

Ce premier cas de figure représente l'exacte antithèse de l'alliance jebero : des relations plus ou moins secrètes, la naissance d'enfants en dehors d'une relation matrimoniale reconnue par les familles et l'abandon de la femme. Cette forme d'union sans vie commune constitue la principale hantise des parents lorsque leur fille atteint l'âge du mariage. Or ces relations avortées entre membres de moitiés distinctes placent d'emblée l'alliance inter-moitié sous le signe de la séduction abusive : les parents de la jeune femme accusent régulièrement ces " séducteurs » d'avoir jeté un sort à leur fille, qui ne pouvait dès lors refuser leurs avances.

À ce premier lien entre l'homme chauve-souris et les membres de l'autre moitié s'en ajoute un autre : l'incapacité de l'homme chauve-souris à rapporter à son épouse de quoi se nourrir s'apparente également à l'attitude de ces jeunes hommes qui, en refusant de vivre avec la mère de leurs enfants, échappent à leurs obligations de chef de famille. Ils n'ouvrent pas de jardins, ni ne partent à la chasse au bénéfice de leur compagne délaissée. Sous cet angle, ces séductions aboutissent à des relations improductives, en contradiction flagrante avec les règles implicites de la co-résidence.

En dehors de ces unions de jeunesse, les alliances matrimoniales inter-moitiés concernent le plus souvent des personnes d'âge mûr se résignant à choisir leur conjoint dans la moitié opposée suite à l'échec successif de précédents mariages au sein de leur propre moitié ou à la mort d'un premier conjoint. Fidel Lomas Chota représentait un troisième cas de figure : longtemps célibataire, il avait épousé une jeune femme de la moitié haute, de 15 ans sa cadette. La mère de son épouse justifiait son accord à ce mariage par le fait qu'elle n'avait plus de " parents» au sein de sa propre moitié (son mari était lui-même d'origine extérieure au village) et ne trouvait donc pas d'autres prétendants pour sa fille. D'une certaine manière, sa situation se rapprochait de celle de la mère de l'épouse de l'homme chauve-souris : ne pouvant fournir au sein de sa propre parentèle des conjoints possibles à sa fille, elle dut se résoudre à cette alliance avec un gendre plus distant.

Séductions de jeunesse ou derniers choix, la grande majorité des mariages inter-moitiés ont donc la particularité de se placer régulièrement en deçà ou au-delà de l'union matrimoniale normale. Ce qui peut dès lors être perçu comme une véritable réticence envers les mariages inter-moitiés se justifie, selon 
les Jebero, par la récurrence des conflits générés par ce type d'union. Ainsi, l'époux qui quitte sa moitié pour venir partager le quotidien de ses beaux-parents se verra notamment jugé responsable de la fuite des animaux hors de leur territoire de chasse. Cela soit du fait d'une malveillance délibérée (le gendre sera accusé d'avoir sciemment jeté un mauvais sort), soit du fait de sa condition même d'« étranger » : l'esprit de la forêt, maître des animaux (sacha ruma ${ }^{9}$ ), traitera le nouveau venu comme un inconnu et refusera de lâcher sa progéniture sur le chemin des chasseurs.

Des échecs successifs lors d'expéditions de chasse peuvent ainsi aboutir à des conflits ouverts entre le gendre et la famille de sa femme, provoquant finalement la dissolution du couple. Il ressortait de mon premier recensement que plus d'un tiers des mariages inter-moitiés s'étaient terminés par des divorces. Les Jebero ne précisent guère les raisons ayant motivé ces ruptures. Il apparaît néanmoins clairement dans leurs propos que les tensions ne concernaient pas directement le couple lui-même, mais bien les familles alliées : " elles ne s'entendaient pas ". Cette incompréhension mutuelle, déjà relevée dans le mythe de l'homme chauvesouris, débouche sur le retour du gendre dans sa moitié d'origine.

L'affection des époux peut cependant les amener à choisir une autre option : l'exil. Le départ du couple permet d'apaiser les tensions familiales. Les époux ne reviennent le plus souvent qu'à la mort de leurs parents respectifs. L'alliance entre les deux familles, rejetée à sa naissance, est elle-même à ce stade définitivement enterrée. Fidel Lomas Chota donne l'exemple de son grand-père :

Mon grand-père, Fidel Chota Chota, était un séducteur. Il a épousé une femme de la moitié haute. Mais les deux familles ne s'entendaient pas. Alors, mon grand-père a dit un jour à sa femme : « mieux vaut nous en aller d'ici ». Il a vécu de nombreuses années en dehors du village et, puis, est revenu faire sa maison là où je vis actuellement.

Le choix du grand-père de Fidel d'établir sa nouvelle demeure au sein de sa propre moitié, en contradiction flagrante avec le principe d'uxorilocalité, renvoie en fait à une pratique extrêmement courante dans le cadre des mariages intermoitiés : la femme part vivre chez son mari.

Les Jebero apportent une double justification : tout d'abord, l'impossibilité pratique dans laquelle se trouve un chasseur d'obtenir des proies du sacha runa sur le territoire de la moitié adverse. Fidel Lomas qui, comme son grand-père, avait fait venir sa jeune femme chez lui justifiait son choix de la manière suivante :

- Lorsque je vais chasser dans la zone de mon beau-père, je reviens sans rien.

- Parce que tu ne connais pas bien cette zone?

- Non. Ce n'est pas ça. Je vais te raconter ce qui m'est arrivé une fois. Je chassais avec mon beau-père. J'avais trouvé un arbre qui donnait beaucoup de fruits. Il y avait de très nombreuses traces de majaz (Cuniculus paca). Les fruits au sol étaient entamés. Les majaz venaient régulièrement manger ces fruits. Alors, je fais ma chapana ${ }^{10}$ et $\mathrm{j}$ 'attends. J'ai attendu toute la nuit et aucun majaz n'est venu. Le sacha runa se méfiait de moi. 
C'est toujours ainsi lorsqu'il ne te connaît pas. C'est pour ça que je ne vais plus chasser chez mon beau-père.

L'explication donnée par Fidel pour justifier son refus d'aller vivre chez son beau-père s'avère symétrique des accusations portées par les parents des épouses : la vie avec des affins trop distants rendrait très improbable l'obtention de nourriture en provenance de la forêt.

Nous retrouvons là, au niveau alimentaire, le principe déjà aperçu dans le mythe d'origine sur le mode de la confiance : la co-résidence avec ses affins est, dans la pratique, estimée impossible. Ses deux aspects sont en fait étroitement liés : comme je l'ai signalé, la confiance ne résulte pas chez les Jebero d'un constat objectif de liens de parenté, mais d'un processus progressif au sein duquel l'entraide régulière et la consommation commune de nourritures et de boissons permettent la création d'un climat de confiance indispensable à la définition d'un champ de parenté. Or le refus du sacha runa de lâcher ses enfants possède une implication évidente qui rejaillit directement sur les conditions de réalisation de ce processus : ce qui ne peut être obtenu ne peut être partagé. Dans un tel contexte, l'affin a toutes les peines du monde à construire avec les parents de sa femme ces liens de confiance, préalables nécessaires à son assimilation comme « parent ».

On comprend dès lors pourquoi ceux-ci choisissent en majorité d'y renoncer et restent avec leur épouse dans leur propre moitié. La virilocalité permet d'éviter cette période de co-résidence avec leurs beaux-parents et de continuer à chasser sur leur propre territoire. Ce faisant, cependant, ils font bien plus que renoncer à créer des liens de parenté avec la famille de leur conjointe. Ramener sa femme chez ses propres consanguins sans rendre aucun service à son beau-père équivaut à une captation à peine voilée. Un comportement prédateur qui rapproche leurs auteurs d'une autre incarnation de l'affinité donnée dans la tradition orale : celle de l'homme-guêpe.

\section{LA GUÊPE OU L'ALLIÉ PRÉDATEUR}

Le mythe de l'homme-guêpe nous permet de mettre en évidence le lien direct perçu par les Jebero entre la pratique virilocale et le thème de la prédation :

Autrefois, la guêpe était un homme. Il allait de maison en maison pour demander la main d'une femme. Une famille accepta de donner sa fille à l'homme. Celui-ci l'emmena directement au cœur de la forêt, à son tambo ${ }^{11}$. Là-bas, il n'y avait rien à manger. L'homme-guêpe avait très faim. Il décida alors de tuer et de manger sa femme.

Il revint ensuite de la forêt et s'en alla chercher une nouvelle épouse. Une nouvelle famille lui donna sa fille qu'il emmena également. Celle-ci disparut comme la première. Les gens commencèrent à se demander : "pourquoi ses femmes disparaissent- 
elles? ». Mais l'homme parvint à obtenir la main d'une troisième femme. Comme d'habitude, il l'emmena immédiatement en forêt. Ils arrivèrent jusqu'à son tambo. À l'aube, l'homme dit à la femme : « femme, reste ici. Je vais aller chasser pour rapporter quelque chose à manger. Attends-moi en préparant le manioc. Je vais apporter un mono negro (Pithecia monachus) et sa progéniture. Vers midi, je serai ici. Pendant ce temps si tu as faim, ne va pas à la rivière. N'attrape pas de crevettes et surtout, là où il y a un grand trou, ne va pas mettre la main ". Alors, il partit chasser.

Mais la femme sentit une grande faim. Elle décida de descendre à la rivière pour attraper des crevettes. De là, elle aperçut un trou d'où elle entendait des bruits sourds. Elle se dit : « ce doit être un grand poisson. Je vais y mettre ma main pour l'attraper ». Ainsi faisant, elle sentit soudain quelque chose qu'elle tira à elle. Apparurent de longs cheveux. Elle tira plus avant et surgit une tête de femme. La femme comprit : " c'est ainsi qu'il fait disparaître ses femmes, mais il ne va pas réussir à me tuer ».

Lorsqu'elle entendit le cri du mono negro, elle courut se cacher à peu de distance du tambo. L'homme arriva, prêt à attraper sa femme. "Où es-tu, femme ? Apparais ! ». Comme personne ne répondait, il se dit : « elle a dû se rendre à la rivière malgré ce que je lui ai dit et a trouvé la tête. Elle s'est échappée maintenant. J'aurais dû la manger hier ». Il ouvrit la marmite et vit le manioc cuit : " que vais-je manger d'autre? ». Il pris sa machette, saisit son mollet et commença à découper sa chair. Il la fit bien cuire et mangea sa propre chair.

Pendant ce temps, la femme continuait d'observer de loin. L'homme avait encore faim et découpa son autre mollet qu'il cuisina et mangea de la même manière. Une fois fini, il se leva pour voir s'il pouvait toujours marcher. Comme il le pouvait, il décida de découper encore un peu de viande. Finalement, lorsqu'il se releva, il s'écroula : il ne pouvait plus marcher.

La femme choisit ce moment pour réapparaître :

- J'ai vu comment tu tuais tes femmes. Tu voulais faire ainsi avec moi.

- Viens ici, tout près, femme.

Mais celle-ci ne s'approcha pas. Elle retourna chez ses frères. «Que faire? » leur demanda-t-elle.

Ses frères dirent : «allons le voir ». Ils arrivèrent au tambo. L'homme était assis. Ils décidèrent de le brûler. Mais, lorsque le corps de l'homme commença à brûler, de nombreuses guêpes jaillirent de son corps et s'enfuirent. C'est pour cela que maintenant les guêpes nous piquent et nous mordent. (Rozenda Maca Lomas).

Ce récit dévoile la dimension potentiellement prédatrice attachée au fait de prendre femme. Dans la société jebero, cette dimension est habituellement effacée au profit d'une logique de don et contre-don. Comme l'indique le mythe, une famille « donne » sa fille. Le principe d'uxorilocalité pose ensuite les conditions d'une "rétribution » de la part du gendre qui, en compensation du don de la femme, doit « rendre » service à son beau-père au quotidien à travers les multiples actes d'entraide qu'implique la vie commune. Or l'homme-guêpe déroge à chaque fois à cette logique. L'accord de la famille à peine obtenu, il emmène immédiatement sa femme à son tambo perdu en pleine forêt. Il refuse dès lors clairement d'attribuer toute compensation au don qu'il vient de recevoir. 
Cette pratique virilocale instaurée par l'homme-guêpe, plus encore que la paresse démontrée parl'homme chauve-souris, enlève à l'alliance entre l'homme et sa belle famille tout fondement empirique : les familles n'arrêtent pas de donner (dans une autre version, c'est une seule famille qui donne ses trois filles) sans jamais recevoir en contrepartiel'aide de leur gendre. La virilocalité devient dès lors dans le mythe l'équivalent d'un rapt. Pire encore, un véritable acte de cannibalisme : l'homme-guêpe ne se contente pas de ramener ses femmes chez lui, il les mange.

Le mariage virilocal transforme donc une possibilité d'alliance en un fait de prédation dont le mythe dénonce toute l'horreur, mais aussi toute la vacuité : la voracité de l'homme-guêpe, qui cherche toujours à prendre sans donner, finit par l'auto-détruire. Être asocial (cannibalisme) et prédateur (virilocalité), cet affin prend surtout le visage d'un ennemi qu'il faut combattre : les frères de la troisième femme, après avoir pris connaissance des pratiques cannibales de leur beau-frère, n'ont pas d'autre choix que de le tuer.

L'homme-guêpe constitue une variante de l'affinité incarnée par l'homme chauve-souris. Ce dernier, séducteur, paresseux et sorcier, figure l'ambiguïté ressentie à l'égard de tout affin détaché de la sphère des parents. Il suscite la méfiance et l'incompréhension. L'homme-guêpe évoque quant à lui la figure du prédateur. Loin de se limiter à un détournement des règles au moyen d'une séduction abusive, il se place ouvertement hors normes. La vie commune, encore formellement possible avec l'homme chauve-souris (son épouse reçoit la visite de sa mère et de ses amies), devient avec lui un affrontement direct.

Ce dernier point est d'ailleurs pleinement assumé par les hommes mariés à des femmes de l'autre moitié, qui y voient une seconde justification à la pratique virilocale. Fernando Lachuma Cachique, qui avait amené chez lui son épouse originaire de la moitié haute, plaçait la virilocalité dans l'optique d'une compétition à laquelle se seraient livrées les deux moitiés :

Auparavant, toute la moitié se réjouissait lorsqu'un homme réussissait à prendre une femme à l'autre moitié. C'était une chose très difficile. L'autre moitié se trouvait ainsi avec une personne de moins. L'autre moitié se réjouissait à son tour lorsque le contraire arrivait : elle récupérait donc le membre perdu.

Les mariages inter-moitiés paraîtraient participer jusqu'à un certain point d'une dynamique de prédation réciproque entre deux groupes ennemis. Une dynamique qui répondrait à la nécessité logique propre à tout système dualiste de créer de l'« Autre », contrepoint indispensable à l'idéal de consanguinité dont j'ai montré la persistance dans les pratiques actuelles (Figure 9). Cette altérité incarnée par la moitié adverse reste cependant bien familière. Les différentes analogies établies ici entre les unions intermoitiés et les figures mythiques de l'homme chauve-souris et de l'homme-guêpe possèdent leurs limites. Plus précisément, la menace potentiellement matérialisée par l'alliance inter-moitié se trouve traditionnellement annihilée par deux facteurs décisifs. 


\begin{tabular}{|c|c|c|}
\hline \multirow[t]{2}{*}{ Parent } & \multicolumn{2}{|c|}{ Ennemi } \\
\hline & Proche & Lointain \\
\hline Frère & Chauve-souris & Guêpe \\
\hline Co-résidence & Voisinage & Extériorité \\
\hline Uxorilocalité & Virilocalité & Virilocalité \\
\hline Don & Séduction & Prédation \\
\hline Entraide & Sorcellerie & Cannibalisme \\
\hline Confiance & Soupcon & Conflit \\
\hline
\end{tabular}

Fig. 9 - Les visages de l'aflinité.

Le premier tient à la nature même de l'opposition sur laquelle se serait construit à l'origine un tel dualisme. Selon la tradition orale jebero, ce dernier résulterait directement de l'impact de la conquête espagnole : en réaction à la domination outrancière d'un Espagnol dénommé significativement "le séducteur » (aputek), certains ancêtres jebero prirent la décision de le tuer, rompant ainsi sciemment avec leurs lois originelles prônant la non-violence. Une autre partie s'y refusa et, devant la fureur manifestée par leurs pairs, choisit de quitter durant un temps le village pour une ville espagnole. Ils ne revinrent qu'une fois christianisés pour former la moitié basse du village, les «meurtriers » de l'Espagnol occupant la moitié haute.

Cette représentation mythique de la naissance du dualisme reprend certains aspects des conditions ayant historiquement présidé à la séparation du village sous l'égide des Jésuites. On se rappelle que ce dualisme est le résultat d'une réorganisation progressive de la mission du fait de l'affluence d'Indiens membres d'autres ethnies. La moitié basse avait fini par constituer le lieu de rassemblement et de métissage d'Indiens aux origines ethniques des plus diverses. Leur arrivée s'inscrivait bien dans un contexte de christianisation de la société jebero. La tradition orale modifie cependant cette version de l'histoire sur un point crucial : ce dualisme serait né de la séparation d'un seul et même peuple. Les Jebero nient l'existence de cette diversité ethnique à l'origine de leur dualisme. Si les habitants de la moitié basse sont présentés dans le mythe comme récemment christianisés, ils restent identifiés comme Jebero. Ils ne sont pour leurs voisins qu'une forme d'altérité minimale, une version différente de soi-même. Comme je l'ai montré ailleurs (Julou 2000), les deux moitiés du village constituent, dans la tradition orale, deux pôles hostiles mais complémentaires. Les habitants de la moitié haute ont besoin de Jebero christianisés pour retrouver les principes fondateurs de leur socialité et reconstituer un monde refermé sur lui-même. À l'inverse, le retour au 
village permet aux habitants de la moitié basse de ne pas se diluer définitivement dans le monde extérieur. À travers leur tradition orale, les Jebero reconnaissent l'altération de leur identité au contact du monde espagnol, mais l'incorporent pour reconstruire une identité bipolaire qui dès lors se suffìt à elle-même.

Le second facteur censé annihiler la dimension « étrangère » des habitants de l'autre moitié réside dans la religion chrétienne elle-même. Depuis près de quatre siècles maintenant, celle-ci diffuse au sein du village des valeurs communes rituellement entretenues tout au long de l'année. À Jeberos comme dans toutes les anciennes missions jésuites, l'église a longtemps été placée au centre du village et monopolisait l'expression officielle du sentiment religieux. Elle constituait le principal pôle d'attraction du village. Autrefois, disent les Jebero, l'église marquait à la fois la frontière entre les territoires des deux moitiés et l'unique endroit où leurs membres étaient amenés à se côtoyer. Lieu de culte et espace médiateur, l'église unissait devant Dieu des moitiés séparées dans la vie de tous les jours, favorisant la création, sous une forme analogique, d'une parenté entre les membres de moitiés adverses. Surtout, elle apportait un contenu à cette identité jebero : celle propre à une communauté de chrétiens, idéalement fondée sur la générosité et la non-violence.

En ce sens, le christianisme a contribué de manière déterminante à encadrer l'opposition dualiste et à la réduire par là même à sa dimension symbolique. $\mathrm{Si}$, comme nous l'avons vu, l'adhésion commune à la religion chrétienne n'empêche pas la suspicion mutuelle et les mésententes, le partage ponctuel d'une même ritualité a longtemps rendu impossible la perception d'un membre du village comme ennemi à part entière, dans toute sa dimension prédatrice. Or ici se situe sans doute le véritable changement dont se font écho les Jebero. Cette forme de dualisme en tant que mode de construction d'une altérité sociale isomorphe permettant idéalement un certain renfermement sur soi-même se voit aujourd'hui supplantée par un phénomène nouveau, qui rompt avec cet équilibre en rendant cet ennemi de plus en plus irréductible à un parent.

\section{L'ENNEMI INTÉRIEUR}

Ce phénomène est lié essentiellement, dans un premier temps, à la forte et soudaine immigration d'une population très diverse au sein du village jebero à l'époque du barbasco: patrons accréditeurs, commerçants, conducteurs de camions, mécaniciens ou simples travailleurs manuels, pour la plupart métis de différentes origines géographiques et sociales, mais également Indiens cocamilla et chayahuita. Autant d'incarnations de l'altérité appelées du jour au lendemain à devenir des co-résidents et bientôt des « parents ».

Les Jebero s'en font l'écho de diverses manières. Certains comme Francisco Inuma Chota insistent sur l'ampleur du mouvement migratoire : "Au temps du barbasco, 1000 hommes vinrent de l'extérieur travailler comme "peones" à 
Jeberos. La majorité d'entre eux formèrent ici une famille et repartirent lorsque leurs enfants furent assez grands. Tous sont partis ».

Sans doute exagérée, cette image d'une multitude anonyme surgissant soudain dans le village, puis s'en allant aussi brusquement en emportant femmes et enfants, tranche significativement avec la représentation habituelle de l'affinité, idéalement inscrite au sein d'un champ de parenté clairement identifié. Comme nous l'avons vu, les Jebero aiment entretenir des relations avec des affins dont ils peuvent justifier de relations d'alliances antérieures. Or les propos de Francisco mettent en évidence l'incompatibilité implicite ressentie par les Jebero entre cette immigration soudaine et la nécessaire identification de tout affīn à un parent classificatoire. Le processus progressif d'effacement de l'altérité de l'affĩn, débordé par la masse des nouveaux arrivants, était rendu inopérant. Surtout, leur départ aussi soudain enlevait toute signification aux relations d'affinité instaurées avec les familles de leurs épouses. Ces affïns en transit, emportant ainsi leur femme loin de leur famille, récupéraient du même coup leur statut d'altérité prédatrice.

D'autres Jebero portent un regard encore plus désabusé sur l'attitude de ces hommes dont la présence fugace n'était motivée que par le potentiel économique offert un temps par les racines de barbasco. Nombre d'entre eux se seraient contentés d'abuser de l'innocence des femmes jebero, pour ensuite les abandonner; elles et leurs enfants. Ces mères étaient alors obligées d'éduquer seules leurs enfants, tout en étant confrontées aux regards désapprobateurs des autres Jebero. Mal à l'aise, Karla Saraza Chota se limitait à ces propos laconiques : « Ma mère a été une femme trompée. Mon père est d'Iquitos, mais je ne l'ai jamais connu. Il est parti, juste comme ça ».

Au vu des analyses qui précèdent, les témoignages de Francisco et Karla rappellent des situations qui nous sont maintenant familières : l'image laissée par ces hommes alterne entre une séduction abusive (chauve-souris) et un rapt généralisé (guêpe). En l'espace de quelques années, ces nouveaux « alliés » ont soudainement incarné la double figure de l'ennemi au sein même du village. Or cette incarnation n'a pas été aussi fugitive que le laisse entendre Francisco. Une quantité non négligeable de ces patrons, commerçants et professeurs, a choisi de rester. Leurs comportements et surtout leur mainmise sur le pouvoir économique et politique du village ont contribué dès lors à modifier en profondeur les relations entre alliés.

Les commerçants et professeurs métis mariés à des femmes jebero se caractérisent, pour la majorité d'entre eux, par leur rejet ostentatoire des implications sociales liées à leur statut d'affin. Ce rejet s'exprime sous différentes formes : justifiant de leur activité professionnelle ou de leur méconnaissance de la forêt, ils ne participent qu'exceptionnellement aux expéditions de chasse ou aux travaux collectifs organisés par les parents de leur épouse, activités pourtant indispensables à leur assimilation comme parents " proches».

Leur relative aisance financière offre les moyens de cette rupture de co-résidence avec les beaux-parents. Ils ne sont pas directement dépendants des 
ressources de l'environnement pour subvenir à leur besoin. Ne se rendant jamais en forêt pour chasser ou pêcher, ils achètent le gibier et les poissons que proposent les Jebero. Cette indépendance économique permet également de se passer des services de leurs affins jebero. Ils vont souvent faire appel, pour l'ouverture de leur espace de jardin comme pour leur entretien, à des hommes et des femmes auxquels ils ont préalablement avancé de l'argent ou fourni gratuitement des biens de consommation courants.

Cette reproduction à l'échelle familiale d'une logique de l'habilitation autrefois propre aux relations avec les patrons favorise, aux yeux des Jebero, leur identification aux commerçants de l'époque du barbasco, dont beaucoup sont d'ailleurs les descendants directs. Comme eux, ces affins métis substituent aux relations de parenté, instaurées sur la logique du don, des rapports d'ordre économique fondés sur la logique de la dette. Ce faisant, ils renforcent leur statut d'altérité au lieu de l'atténuer.

Ce désengagement social trouve également son expression dans l'espace. Les propriétaires de commerce comme les fonctionnaires ne construisent pas leur demeure à proximité immédiate de celle des parents de leur femme, mais optent le plus souvent pour acheter une maison déjà construite le plus près possible du centre du village.

Il faut s'arrêter un instant sur ce choix, car il est parfaitement révélateur des transformations vécues par la société jebero au cours des dernières décennies. Le centre du village s'est vu effectivement profondément reconfiguré suite à l'incendie qui frappa l'ancienne église dans les années 1960. Comme je l'ai indiqué, celle-ci était, depuis l'époque coloniale, l'axe de référence absolu pour les habitants du village. La mairie prit pourtant la décision de construire sur ses cendres une Place d'Armes, avec un monument érigé en son centre, peint aux couleurs du drapeau national. L'église devint à cette date un simple élément constitutif du centre, au même titre que la mairie, le bureau du gouverneur ou le centre d'administration de l'école. La décision du maire n'était pas anodine : ancienne mission chrétienne autrefois prise en modèle, Jeberos se présentait comme une version modeste, encore en devenir, des villes péruviennes. Une métamorphose symbolique du passage, vécu par les Jebero, d'une communauté villageoise d'Indiens chrétiens, avec l'église comme référence centrale, à une communauté de paysans métis prenant comme modèle la nation péruvienne.

Si l'attraction vers ce centre métamorphosé restait limitée aux patrons et commerçants métis, on pourrait conclure à une simple confrontation, au sein même du village, entre deux modèles sociologiques, comme ce fut sans doute encore le cas à l'époque du barbasco. Aujourd'hui cependant, une part non négligeable de Jebero acquiert des emplois rémunérés et opte pour des comportements similaires. Les Jebero choisissent de faire construire ou d'acheter une maison à proximité de la Place d'Armes. Au-delà de ces choix individuels, on assiste plus fondamentalement à la formulation d'un nouveau dualisme de type 
concentrique tendant à distinguer le centre du village, dédié à la gestion administrative, aux prises de décision politique et au commerce, et caractérisé par la relative aisance financière de ses habitants, et sa périphérie, habitée par une majorité de Jebero se limitant à des activités traditionnelles très peu rémunératrices. Cette distinction, en réalité graduelle mais bien visible, révèle la suprématie actuelle de nouveaux codes et valeurs issus de la hiérarchie économique et du niveau scolaire, dont l'impact s'avère décisif sur les modalités d'alliances matrimoniales pratiquées au sein du village.

Une telle redistribution de l'espace villageois ne renvoie pas à une opposition d'ordre ethnique. Elle ne distingue pas une zone centrale métisse d'une zone périphérique réservée aux Jebero, derniers représentants de l'ethnie et farouches défenseurs de l'endogamie locale et des traditions. Les Jebero de la périphérie ne sont pas exempts de métissage. Celui-ci est simplement différent et révèle à mon sens la suprématie actuelle de principes distinctifs d'ordre socio-économique : lorsqu'ils ne trouvent pas de conjoints au sein du village, les Jebero du centre choisissent régulièrement d'épouser des femmes ou des hommes métis originaires des principales villes (Yurimaguas ou Iquitos) de la région, alors que ceux de la périphérie du village optent pour des unions avec des membres des communautés avoisinantes (Chayahuita, Cocamilla, métis pauvres du Marañon).

Ces mariages rapprochent des personnes aux dispositions économiques globalement équivalentes. Si le principe fondateur des choix matrimoniaux jebero mis en évidence précédemment reste pertinent (les époux partagent un mode de vie suffisamment proche pour envisager une union durable), les codes définissant cette notion de proximité ont été profondément renouvelés : l'origine géographique ou ethnique, l'existence ou l'absence préalable de vie commune ne sont plus des critères déterminants. Tend à primer désormais la plus ou moins grande familiarité des deux conjoints avec le mode de vie des villes métisses pris aujourd'hui comme modèle. Or l'adoption de ce modèle implique une conception différente des relations d'affinité : le choix de la localisation de sa demeure est implicitement lié à la position économique occupée par l'époux. Ce faisant, ce dernier rompt souvent avec le principe d'uxorilocalité qui était autrefois la règle dans le choix de la résidence du couple. Les dérogations au principe de co-résidence ont tendance à se multiplier et aboutissent à désolidariser les notions d'alliance et de vie partagée, synonyme de parenté. Leur équivalence implicite, autrefois la norme, n'est plus aussi pertinente. L'affinn, échappant à son assimilation à un parent consanguin, maintient sa distance et rend tangible son altérité.

Cette transformation du rapport à l'affininité porte en germe les tensions familiales décrites en introduction. Contrairement à l'image idéalisée du village des ancêtres comme communauté de parents aux relations harmonieuses, le village jebero actuel se perçoit de plus en plus comme un conglomérat de gens s'affirmant comme mutuellement différents. Or, comme nous l'avons vu avec l'homme chauve-souris, ces différences ouvrent une brèche dans la confiance 
attribuée aux alliés. Ces derniers se voient dès lors potentiellement soupçonnés de tous les défauts propres à cette figure de l'affinité : ils seraient paresseux, jaloux et sorciers.

De tels soupçons, autrefois annihilés par le biais d'une ritualité chrétienne apaisante, s'inscrivent dans un contexte nouveau qui va en accentuer les conséquences délétères : chaque famille tend aujourd'hui à construire sa propre stratégie de développement économique, distincte de celle des autres Jebero, y compris de leurs propres parents. Ce qui n'était encore qu'une prise de distance va dès lors déboucher sur une généralisation des accusations mutuelles.

L'exemple du père d'Abraham Chota, cité en introduction, est à cet égard tout à fait emblématique : peu avant son ensorcellement, le père d'Abraham venait d'acquérir trois vaches avec l'espoir de constituer à l'avenir un élevage fructueux. Seules quelques familles réussirent à réunir le pécule nécessaire à l'achat des premières têtes de bétail. Premières qui furent bien souvent les dernières. Ce type d'élevage rencontra de multiples difficultés pour s'instaurer durablement. L'obstacle le plus déterminant ne fut pas le milieu écologique, peu amène, mais les tensions sociales que la présence du bétail ne manquait pas de provoquer. Les vaches, aux dires des Jebero, s'échappaient régulièrement de leurs enclos, voire n'y étaient parfois pas enfermées, et ne cessaient de déranger les voisins de leurs propriétaires en s'introduisant dans les maisons, brisant les marmites ou piétinant les jardins. Poussés par la colère, les Jebero lésés finirent par empoisonner ces vandales en leur faisant boire du barbasco. L'élevage bovin disparut très vite des alentours du village. Colère, mais peut-être aussi ressentiment personnel, symptôme d'une certaine jalousie à l'égard des propriétaires des bêtes. Telle est en tout cas l'explication d'Abraham Chota évoquant le destin funeste de son père ensorcelé et finalement tué par son propre frère, envieux de la réussite de son parent.

Ces accusations de sorcellerie sont devenues monnaie courante dans le village, débouchant même parfois sur le meurtre des sorciers supposés. Sorcellerie et vengeance meurtrière étaient pourtant présentées par la tradition orale comme propres à leurs voisins indigènes. Si certains récits mythiques légitimaient le meurtre ou le rapt (perçus sur un mode défensif) pour repousser l'« Autre » en dehors de ses frontières, la société jebero se considérait jusqu'alors épargnée en son sein même. Or tout le drame est là : ces conflits n'éclatent plus dans des zones limitrophes. Ils concernent des parents consanguins, soit le noyau de la cohésion sociale. L'incrédulité de Miguel devant le diagnostic du chamane se comprend parfaitement : il porte une nouvelle atteinte, la plus grave qui soit, à la confiance entre parents. Ces derniers ne sont plus seulement différents (comme la chauvesouris), mais des ennemis potentiels qu'il faut tuer (comme la guêpe). Si la menace se limitait, à l'aube des temps, à une affinité jivaro radicalement extérieure, les Jebero se sont depuis découvert un ennemi intérieur des plus inattendus : leurs propres « parents ». 


\section{Conclusion}

Les Jebero se sentent aujourd'hui doublement « Autres ». Leur adoption, au cours du $\mathrm{xx}^{\mathrm{e}}$ siècle, d'un modèle économique caractéristique du monde métis a provoqué, selon eux, l'émergence de comportements vindicatifs estimés propres à leurs voisins indigènes. Une double métamorphose, en somme, qui a surtout eu pour conséquence un changement profond de leur conception même de la notion d'affinité : celle-ci, idéalement «invisible », progressivement assimilée à la consanguinité, est devenue une incarnation intime de l'altérité avec laquelle il leur faut désormais cohabiter. L'allié s'intègre tout en maintenant sa différence. Le "parent » est un co-résident qui garde néanmoins ses distances, quitte à être perçu comme un ennemi potentiel. Une révolution des critères de l'alliance qui pourrait donner sens au changement d'attitude adopté récemment par les Jebero dans leurs relations avec la nation péruvienne.

Comme je l'ai précisé en introduction, ceux-ci ont choisi récemment de réaffirmer, comme beaucoup d'autres peuples d'origine amérindienne, leur particularisme identitaire après des décennies d'« invisibilité ». Cette double attitude successive adoptée par les Amérindiens en phase d'intégration, marquée par un effacement de leur différence, puis par sa mise en avant, a trop souvent été perçue et interprétée comme le résultat d'une confrontation : l'invisibilité (autrement dit, le dépouillement de tout signe extérieur de leur indianité) répondant au discours discriminatoire de leurs voisins métis, la démarche revendicative actuelle traduisant pour sa part, et selon les auteurs, une soumission au symbolisme occidental (Conklin 1997), sensible à la visibilité ethnique, ou un « compromis » obtenu de haute lutte (Turner 1991) entre une intégration nécessaire à la nation et une revalorisation légitime de leur identité. Ces options successives me semblent également relever d'un processus endogène dont j'ai essayé de déterminer chez les Jebero la principale caractéristique : l'idéal de l'« allié invisible », qui justifiait aux yeux des Amérindiens souhaitant (ou étant forcés de) s'intégrer à la société nationale leur adoption progressive de l'apparence de cette société, s'est vu aujourd'hui remplacé par un autre idéal, celui de l'affin qui s'intègre tout en manifestant ostensiblement son altérité.

Une évolution qui donne en apparence des résultats positifs (notamment la reconnaissance offícielle d'une certaine diversité culturelle), mais qui, comme le montre l'exemple de la société jebero, possède également des conséquences locales plus délétères : si les Jebero recherchent des relations harmonieuses avec l'extérieur, c'est au prix d'une détérioration sensible de leur propre cohésion sociale. *

* Manuscrit reçu en juillet 2007, accepté pour publication en octobre 2008. 


\section{NOTES}

C'est grâce au Legs Lelong que j'ai pu écrire cet article, je tiens donc à remercier ici les personnes qui m'ont attribué cette bourse.

1. Mon travail de terrain s'est déroulé entre 1996 et 2000 dans le cadre d'une recherche soutenue par l'Institut français d'études andines.

2. La langue, le vêtement ainsi que l'artisanat traditionnel des Jebero ont été abandonnés à cette époque.

3. Sous cet angle, les Jebero reproduisent un schéma bien connu : les auteurs de L'Inca, l'Espagnol et le sauvage insistent dans leur conclusion sur la manière particulière dont nombre de sociétés amérindiennes construisent, au travers d'organisations sociales dualistes ou de guerres intra-tribales, une figure " minimale » de l'altérité leur garantissant une reproduction sociale idéalement autonome : «un trait commun à des sociétés amazoniennes et andines est d'avoir institué en leur sein des partenaires rivaux ou "ennemis", en sorte que l'autre qui fonde une frontière et une identité, est une partie de soi sans cesse opposée à soi-même. [Ainsi,] les organisations dualistes des basses terres comme les systèmes de guerres intra-tribales montrent une certaine tendance à refermer sur soi un ensemble qui trouve son unité et son équilibre dynamique dans la rivalité interne d'éléments isomorphes, mais formellement opposés » (Renard-Casevitz et al. 1986, pp. 357-358). De telles remarques me semblent devoir s'appliquer parfaitement à l'ancien dualisme jebero (tel en tout cas qu'il subsiste dans la mémoire des habitants du village). L'opposition rituelle des moitiés parait bien avoir été corrélative d'un principe d'endogamie locale, d'un "entre soi » idéalement préservé : l'existence d'une figure limitée de l'altérité, représentée par les habitants de l'autre moitié, offrait les conditions d'une reproduction autonome de la vie sociale.

4. Se reporter à l'analyse détaillée de ce mythe dans Julou (2006). Voir également Julou (2000).

5. Les statistiques proposées dans ce texte ont été élaborées à partir d'un recensement de 371 unions matrimoniales (222 pour la moitié haute et 149 pour la moitié basse), étalées sur trois générations.

6. Comme le laisse apparaître la Figure 3, Rozendo avait lui-même épousé sa belle-scur en première noce.

7. L'analyse de Whitten (1976, p. 112) concernant les termes d'adresse employés par les Canelos quichua me paraît parfaitement applicable au cas jebero. Pour ces deux sociétés, " affinal bond of husband and wife merges the statuses of father and mother for the wo spouses, an example of the criterion of affinity in stipulating consanguinity " .

8. Pour des raisons que je développerai plus loin, les mariages inter-moitiés se révèlent très majoritairement virilocaux.

9. Terme d'origine quechua signifiant littéralement « personne de la forêt ».

10. Échaffaudage en bois sur lequel s'installe le chasseur, à près de deux mètres au-dessus du sol.

11. Terme désignant, chez les Jebero, une maison isolée en forêt, distincte de la maison située dans un village.

\section{RÉFÉRENCES CITÉES}

\section{Conklin Beth Ann}

1997 " Body paint, feathers and VCRs : aesthetics and authenticity in Amazonian activism ", American Ethnologist, 24 (4), pp. 711-737.

Gow Peter

1991 Of mixed blood: kinship and history in Peruvian Amazonia, Oxford University Press, Oxford. 
JuLOU Ronan

2000 «Les prédateurs d'Histoire ou la reconstruction du passé par les Indiens jebero ", Bulletin de l'IFEA, 29 (2), Lima, pp. 189-214.

2006 L'autre en soi. Intériorisation de l'altérité et métamorphose identitaire chez les Jebero d'Amazonie péruvienne, thèse de doctorat, École des Hautes Études en Sciences Sociales, Paris.

LÉvi-Strauss Claude

1962 La pensée sauvage, Plon, Paris.

OVERING KaPLAN Joan

1975 The Piaroa, a people of the Orinoco basin : a study in kinship and marriage, Clarendon, Oxford.

Renard-Casevitz France Marie, Thierry Saignes et Anne Christine Taylor

1986 L'Inca, l'Espagnol et les Sauvages, ADPF, coll. « Recherches et Civilisations ", Paris.

Sтоскs Anthony Wayne

1981 Los nativos invisibles. Notas sobre la historia y realidad actual de los cocamilla del rio Huallaga, Centro Amázonico de Antropología y Applicación Práctica (CAAAP), Lima.

TURNER Terence

1991 "Representing, resisting, rethinking : historical transformations of Kayapo culture and anthropological consciousness ", in G. Stocking Jr (ed.), Colonial situations. Essays on the contextualization of ethnographic knowledge, HOA v. 7, The University of Wisconsin Press, Madison, pp. 285-313.

VILACA Aparecida

2002a "Making kin out of others in Amazonia », Man. Journal of the Royal Anthropological Institute, 8, pp. 347-365.

$2002 \mathrm{~b}$ «Missions et conversions chez les Wari' : entre protestantisme et catholicisme ", L'Homme, 164, pp. 57-80.

Viveiros De Castro Eduardo

2001 «GUT feelings about Amazonia : potential aftinity and the construction of sociality ", in Laura Rival and Neil Whitehead (eds), Beyond the visible and the material : the amerindianization of society in the work of Peter Rivière, Oxford University Press, Oxford, pp. 19-43.

2002 A inconstância da alma salvagem - e outros ensaios de antropologia, Cosac \& Naify, São Paulo.

WhitTEN Norman Jr.

1976 Sacha Ruma. Ethnicity and adaptation of the Ecuadorian jungle Quechua, University of Illinois Press, Urbana. 